\title{
Palladium-Catalyzed Carboxylation of Activated Vinylcyclopropanes with $\mathrm{CO}_{2}$
}

Tsuyoshi Mita,* Hiroyuki Tanaka, Yuki Higuchi, and Yoshihiro Sato*

Faculty of Pharmaceutical Sciences, Hokkaido University, Sapporo 060-0812, Japan

tmita@pharm.hokudai.ac.jp

biyo@pharm.hokudai.ac.jp

Supporting Information 


\section{Table of Contents}

(A) General S3

(B) Synthesis of Substrates $\quad$ S3

(C) Palladium-Catalyzed Carboxylation of Vinylcyclopropanes S3

(D) Determination of Relative Configuration $\quad$ S7

(C) ${ }^{1} \mathrm{H}$ and ${ }^{13} \mathrm{C}$ NMR Spectra $\quad \mathrm{S} 10$ 


\section{(A) General}

Infrared (IR) spectra were recorded on a JASCO FT/IR 460 Plus Fourier transform infrared spectrophotometer. NMR spectra were recorded on a JEOL ECA-500 spectrometer, operating at $500 \mathrm{MHz}$ $\left({ }^{1} \mathrm{H}\right), 125 \mathrm{MHz}\left({ }^{13} \mathrm{C}\right)$, or $202 \mathrm{MHz}\left({ }^{31} \mathrm{P}\right)$. Chemical shifts in $\mathrm{CDCl}_{3}$ were reported in the scale relative to $\mathrm{CHCl}_{3}\left(7.26 \mathrm{ppm}\right.$ ) for ${ }^{1} \mathrm{H} \mathrm{NMR}$, to $\mathrm{CDCl}_{3}(77.0 \mathrm{ppm})$ for ${ }^{13} \mathrm{C} \mathrm{NMR}$, and to $\mathrm{P}(\mathrm{O})(\mathrm{OH})_{3}(0 \mathrm{ppm})$ for ${ }^{31} \mathrm{P}$ NMR as internal references. Chemical shifts in $\mathrm{C}_{6} \mathrm{D}_{6}$ were reported in the scale relative to benzene (7.16 ppm) for ${ }^{1} \mathrm{H}$ NMR. ESI mass spectra were measured on a Thermo Scientific Exactive. EI mass spectra were measured on a JEOL JMS-T100GCv. Column chromatography was performed with silica gel Kanto 60 (230-400 mesh ASTM). Dry THF was purified under argon using the Ultimate Solvent System (Glass Counter Inc.). $\mathrm{Pd}(\mathrm{acac})_{2}$ was purchased from Sigma-Aldrich Co. LLC. and used as received. $\mathrm{PPh}_{3}$ was purchased from Kishida Chemical Co., Ltd., and used after recrystallization. $\mathrm{ZnEt}_{2}$ (1 M in hexane) was purchased from Kanto Kagaku, and used as received. A cylinder of $\mathrm{CO}_{2}$ was purchased from Hokkaido Air Water, Inc. In general, reactions were carried out under an argon atmosphere unless otherwise noted.

\section{(B) Synthesis of Substrates}

Vinylcyclopropanes 1a, 1b, 1c, and 1d were prepared according to the reported methods. ${ }^{1}$ 1e was prepared according to the reported method. ${ }^{2} \mathbf{1 f}$ and $\mathbf{1 g}$ were prepared according to the method. ${ }^{3} \mathbf{1 h}$ was synthesized from 3-penten-2-one through cyclopropanation ${ }^{4}$ followed by Wittig reaction ${ }^{3}$ with $\mathrm{Ph}_{3} \mathrm{PCH}_{3} \mathrm{Br}$. 1i was synthesized from cinnamaldehyde through proline-catalyzed cyclopropanation ${ }^{5}$ followed by Wittig reaction ${ }^{3}$ with $\mathrm{Ph}_{3} \mathrm{PCH}_{3} \mathrm{Br}$.

\section{(C) Palladium-Catalyzed Carboxylation of Vinylcyclopropanes}

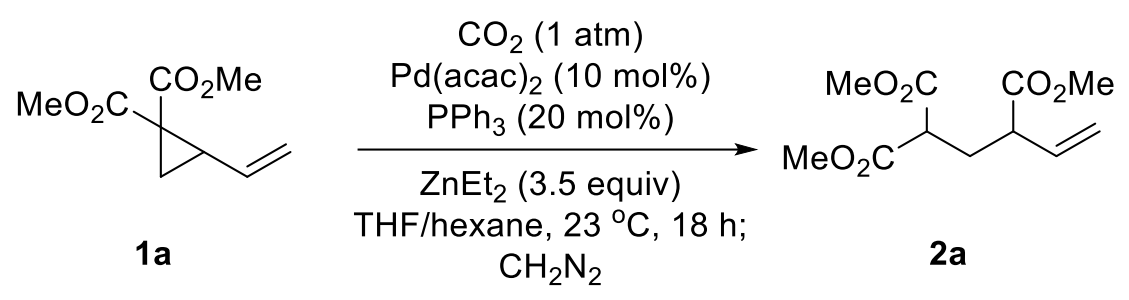

$\mathrm{Pd}(\mathrm{acac})_{2}(6.1 \mathrm{mg}, 0.02 \mathrm{mmol}, 10 \mathrm{~mol} \%)$ and $\mathrm{PPh}_{3}(10.5 \mathrm{mg}, 0.04 \mathrm{mmol}, 20 \mathrm{~mol} \%)$ was placed on the bottom of a test tube containing a stirring bar. The tube was evacuated and refilled with $\mathrm{CO}_{2}$ (balloon) five times. After the addition of dry THF $(0.5 \mathrm{~mL})$, the mixture was stirred at room temperature for 10 min under $\mathrm{CO}_{2}$ atmosphere (1 atm: balloon). Vinylcyclopropane 1a $(36.8 \mathrm{mg}, 0.20 \mathrm{mmol})$, THF (0.5 mL), and $\mathrm{ZnEt}_{2}$ ( $1 \mathrm{M}$ solution in hexane, $0.7 \mathrm{~mL}, 0.70 \mathrm{mmol}, 3.5$ equiv) were added at $0{ }^{\circ} \mathrm{C}$. The resulting reaction mixture was stirred at $23{ }^{\circ} \mathrm{C}$ for $18 \mathrm{~h}$. After cooling to $0{ }^{\circ} \mathrm{C}, \mathrm{HCl}$ aq. $(3 \mathrm{M})$ and water were added, and the mixture $(\mathrm{pH}<2)$ was extracted with AcOEt three times. The combined organic layer was washed with brine and dried over $\mathrm{Na}_{2} \mathrm{SO}_{4}$. After the solvent was removed under reduced pressure, and the residue was diluted with $\mathrm{Et}_{2} \mathrm{O}$ (ca. $4 \mathrm{~mL}$ ) followed by treatment of $\mathrm{CH}_{2} \mathrm{~N}_{2}$, which was prepared from 1-methyl-3-nitro-1-nitrosoguanidine and $40 \%$ aq. KOH. After $10 \mathrm{~min}$, the mixture was directly

1 Dieskau, A. P.; Holzwarth, M. S.; Plietker, B. J. Am. Chem. Soc. 2012, 134, 5048.

2 Zeuner, F.; Angermann, J.; Moszner, N. Synth. Commun. 2006, 36, 3679.

3 Miura, K.; Fujisawa, N.; Hosomi, A. J. Org. Chem. 2004, 69, 2427.

4 Menn, J.-C. L.; Tallec, A.; Sarrazin, J. Can. J. Chem. 1991, 69, 761.

5 Ibrahaem, I.; Zhao, G.-L.; Rios, R.; Vesely, J. Sundén, H.; Dziedric, P.; Córdova, A. Chem. Eur. J. 2008, $14,7867$. 
concentrated under high vacuum to afford the crude product. Yield of 2a was determined at this stage using 1,1,2,2-tetrachloroethane ( $\delta=5.9 \mathrm{ppm}$ in $\mathrm{CDCl}_{3}, 2 \mathrm{H}$ ) as an internal standard (87\% yield). It was then purified by silica gel column chromatography (hexane/AcOEt, 7:1), affording trimethyl pent-4-ene-1,1,3-tricarboxylate (2a) $(42.3 \mathrm{mg}, 0.173 \mathrm{mmol})$ as pale yellow oil in $87 \%$ yield.

Trimethyl pent-4-ene-1,1,3-tricarboxylate (2a)

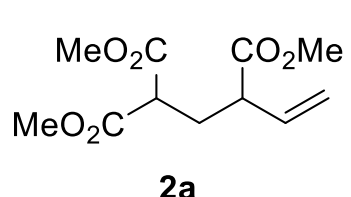

$2 a$

Pale yellow oil; IR (neat): 2956, 1736, 1639, 1438, 1355, 1201, 1160, 1029, 930, $831 \mathrm{~cm}^{-1} ;{ }^{1} \mathrm{H}$ NMR $\left(500 \mathrm{MHz}, \mathrm{CDCl}_{3}\right) \delta=5.75(\mathrm{ddd}, J=17.8,10.5,8.4 \mathrm{~Hz}, 1 \mathrm{H})$, $5.20(\mathrm{~d}, J=10.5 \mathrm{~Hz}, 1 \mathrm{H}), 5.17(\mathrm{~d}, J=17.8 \mathrm{~Hz}, 1 \mathrm{H}), 3.74(\mathrm{~s}, 3 \mathrm{H}), 3.72(\mathrm{~s}, 3 \mathrm{H})$, $3.68(\mathrm{~s}, 3 \mathrm{H}), 3.43(\mathrm{dd}, J=8.0,6.9 \mathrm{~Hz}, 1 \mathrm{H}), 3.11-3.05(\mathrm{~m}, 1 \mathrm{H}), 2.40-2.34(\mathrm{~m}, 1 \mathrm{H})$, 2.18-2.11 (m, 1H); ${ }^{13} \mathrm{C}$ NMR $\left(125 \mathrm{MHz}, \mathrm{CDCl}_{3}\right) \delta=173.2,169.4,169.2,134.3,118.9,52.6,52.1,49.1$, 47.6, 30.3 ppm; HRMS (EI) m/z calcd for $\mathrm{C}_{11} \mathrm{H}_{16} \mathrm{O}_{6}[\mathrm{M}]^{+}:$244.0947. Found: 244.0941.

\section{1,1-Diethyl 3-methylpent-4-ene-1,1,3-tricarboxylate (2b)}

(1b: $42.4 \mathrm{mg}, 0.20 \mathrm{mmol}$; reaction time: $18 \mathrm{~h}$; reaction temperature: $23{ }^{\circ} \mathrm{C}$; The product was purified by silica gel column chromatography (hexane/AcOEt, 7:1) to afford $\mathbf{2 b}(40.7 \mathrm{mg}, 0.150 \mathrm{mmol}$ ) as colorless oil in $75 \%$ yield.)

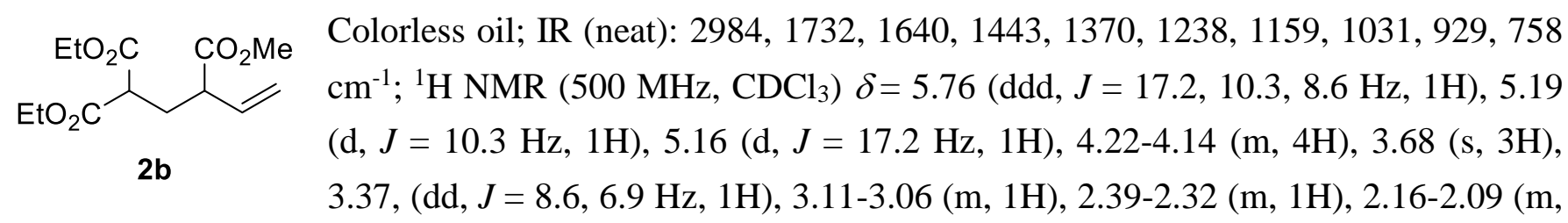

1H), 1.27-1.23 (m, 6H); $\left.{ }^{13} \mathrm{C} \mathrm{NMR} \mathrm{(125} \mathrm{MHz,} \mathrm{CDCl}_{3}\right) \delta=173.2,169.0,168.8,134.4,118.8,61.51,61.49$, 52.0, 49.4, 47.6, 30.2, 14.01, 13.98 ppm; HRMS (EI) m/z calcd for $\mathrm{C}_{13} \mathrm{H}_{20} \mathrm{O}_{6}[\mathrm{M}]^{+}:$272.1260. Found: 272.1265 .

\section{1,1-Dibenzyl 3-methylpent-4-ene-1,1,3-tricarboxylate (2c)}

(1c: $67.3 \mathrm{mg}, 0.20 \mathrm{mmol}$; reaction time: $18 \mathrm{~h}$; reaction temperature: $23{ }^{\circ} \mathrm{C}$; The product was purified by silica gel column chromatography (hexane/AcOEt, 7:1) to afford $2 \mathrm{c}(64.2 \mathrm{mg}, 0.162 \mathrm{mmol})$ as colorless oil in $81 \%$ yield.)

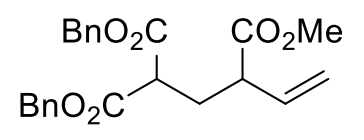

2c

Colorless oil; IR (neat): 3033, 2952, 1732, 1498, 1456, 1378, 1216, 1154, 753, 697 $\mathrm{cm}^{-1} ;{ }^{1} \mathrm{H}$ NMR $\left(500 \mathrm{MHz}, \mathrm{CDCl}_{3}\right) \delta=7.35-7.30(\mathrm{~m}, 6 \mathrm{H}), 7.30-7.27(\mathrm{~m}, 4 \mathrm{H}), 5.74$ (ddd, $J=17.6,9.6,8.9 \mathrm{~Hz}, 1 \mathrm{H}), 5.17-5.07(\mathrm{~m}, 6 \mathrm{H}), 3.66(\mathrm{~s}, 3 \mathrm{H}), 3.52(\mathrm{dd}, J=8.0$, $6.9 \mathrm{~Hz}, 1 \mathrm{H}), 3.10-3.05(\mathrm{~m}, 1 \mathrm{H}), 2.45-2.39(\mathrm{~m}, 1 \mathrm{H}), 2.21-2.15(\mathrm{~m}, 1 \mathrm{H}) ;{ }^{13} \mathrm{C} \mathrm{NMR}$ $\left(125 \mathrm{MHz}_{1} \mathrm{CDCl}_{3}\right) \delta=173.1,168.6,168.4,135.2,135.1,134.2,128.5,128.3,128.2,119.0,67.24,67.22$, 52.0, 49.4, 47.5, 30.2 ppm; HRMS (EI) m/z calcd for $\mathrm{C}_{23} \mathrm{H}_{24} \mathrm{O}_{6}[\mathrm{M}]^{+}:$396.1573. Found: 396.1572.

\section{1,1-Di-tert-butyl 3-methyl pent-4-ene-1,1,3-tricarboxylate (2d)}

(1d: $53.7 \mathrm{mg}, 0.20 \mathrm{mmol}$; reaction time: $18 \mathrm{~h}$; reaction temperature: $23{ }^{\circ} \mathrm{C}$; The product was purified by silica gel column chromatography (hexane/AcOEt, 7:1) to afford $2 \mathbf{d}(59.1 \mathrm{mg}, 0.180 \mathrm{mmol}$ ) as pale yellow oil in $90 \%$ yield.) 


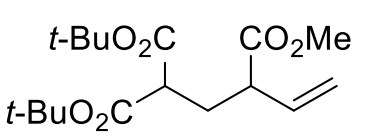

2d

Pale yellow oil; IR (neat): 2979, 1740, 1639, 1393, 1369, 1255, 1164, 926, 848, $757 \mathrm{~cm}^{-1} ;{ }^{1} \mathrm{H}$ NMR $\left(500 \mathrm{MHz}, \mathrm{CDCl}_{3}\right) \delta=5.77(\mathrm{ddd}, J=16.5,9.7,8.7 \mathrm{~Hz}, 1 \mathrm{H})$, $5.19(\mathrm{~d}, J=9.7 \mathrm{~Hz}, 1 \mathrm{H}), 5.16(\mathrm{~d}, J=16.5 \mathrm{~Hz}, 1 \mathrm{H}), 3.69(\mathrm{~s}, 3 \mathrm{H}), 3.18(\mathrm{dd}, J=8.0$, $6.9 \mathrm{~Hz}, 1 \mathrm{H}), 3.12-3.06(\mathrm{~m}, 1 \mathrm{H}), 2.30-2.24(\mathrm{~m}, 1 \mathrm{H}), 2.06-2.00(\mathrm{~m}, 1 \mathrm{H}), 1.46(\mathrm{~s}$, 9H), 1.45 (s, 9H); ${ }^{13} \mathrm{C}$ NMR (125 MHz, $\left.\mathrm{CDCl}_{3}\right) \delta=173.5,168.4,168.1,134.7,118.6,81.7,81.6,52.0$, 51.3, 47.7, 30.3, 27.90, 27.87 ppm; HRMS (EI) m/z calcd for $\mathrm{C}_{17} \mathrm{H}_{28} \mathrm{O}_{6}[\mathrm{M}-\mathrm{O} t-\mathrm{Bu}]^{+}:$255.1232. Found: 255.1229 .

1-Ethyl 5-methyl 2-(diethoxyphosphoryl)-4-vinylpentanedioate (2e)

(1e: $55.3 \mathrm{mg}, 0.20 \mathrm{mmol}, \mathrm{dr}=5.5: 1$; reaction time: $18 \mathrm{~h}$; reaction temperature: $23{ }^{\circ} \mathrm{C}$; The product was purified by silica gel column chromatography (hexane/AcOEt, 7:1 1:1) to afford 2e (27.0 mg, 0.080 mmol) as colorless oil in $40 \%$ yield; $\mathrm{dr}=1: 1$.)

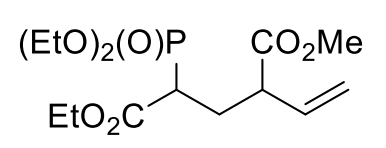

$2 e$

Colorless oil; IR (neat): 2984, 1376, 1639, 1442, 1369, 1246, 1159, 1050, 1024, $972 \mathrm{~cm}^{-1} ;{ }^{1} \mathrm{H}$ NMR (diastereomixture: $\left.500 \mathrm{MHz}, \mathrm{CDCl}_{3}\right) \delta=5.80-5.68(\mathrm{~m}, 1 \mathrm{H}+$ $1 \mathrm{H}), 5.24-5.11(\mathrm{~m}, 2 \mathrm{H}+2 \mathrm{H}), 4.24-4.09(\mathrm{~m}, 6 \mathrm{H}+6 \mathrm{H}), 3.682(\mathrm{~s}, 3 \mathrm{H}), 3.680(\mathrm{~s}$, $3 \mathrm{H}), 3.14-2.93(\mathrm{~m}, 2 \mathrm{H}+2 \mathrm{H}), 2.45-2.04(\mathrm{~m}, 2 \mathrm{H}+2 \mathrm{H}), 1.35-1.24(\mathrm{~m}, 9 \mathrm{H}+9 \mathrm{H})$;

${ }^{13} \mathrm{C}$ NMR (diastereomixture: $\left.125 \mathrm{MHz}, \mathrm{CDCl}_{3}\right) \delta=173.2,173.1,168.7\left(J_{C-P}=4.8 \mathrm{~Hz}\right), 168.5\left(J_{C-P}=4.8\right.$ $\mathrm{Hz}), 134.6,134.0,119.6,118.4,62.91\left(J_{C-P}=6.0 \mathrm{~Hz}\right), 62.88\left(J_{C-P}=7.7 \mathrm{~Hz}\right), 62.8\left(J_{C-P}=7.2 \mathrm{~Hz}\right), 62.7$ $\left(J_{C-P}=6.0 \mathrm{~Hz}\right), 61.52,61.49,52.1,52.0,48.5\left(J_{C-P}=14.4 \mathrm{~Hz}\right), 48.3\left(J_{C-P}=15.6 \mathrm{~Hz}\right), 43.3\left(J_{C-P}=130.8\right.$ $\mathrm{Hz}), 42.8\left(J_{C-P}=132.0 \mathrm{~Hz}\right), 28.8\left(J_{C-P}=3.6 \mathrm{~Hz}\right), 28.3\left(J_{C-P}=2.4 \mathrm{~Hz}\right), 16.34\left(J_{C-P}=6.0 \mathrm{~Hz}\right), 16.30\left(J_{C-P}=\right.$ $6.0 \mathrm{~Hz}$ ), 14.1, 14.0; ${ }^{31} \mathrm{P} \mathrm{NMR}$ (diastereomixture: $202 \mathrm{MHz}, \mathrm{CDCl}_{3}$ ) $\delta=24.8,24.5 \mathrm{ppm}$; HRMS (EI) m/z calcd for $\mathrm{C}_{14} \mathrm{H}_{25} \mathrm{O}_{7} \mathrm{P}[\mathrm{M}]^{+}:$336.1338. Found: 336.1326 .

Trimethyl 4-methylpent-4-ene-1,1,3-tricarboxylate (2f)

(1f: $39.6 \mathrm{mg}, 0.20 \mathrm{mmol}$; reaction time: $18 \mathrm{~h}$; reaction temperature: $23{ }^{\circ} \mathrm{C}$; The product was purified by silica gel column chromatography (hexane/AcOEt, 7:1) to afford $\mathbf{2 f}$ (40.3 $\mathrm{mg}, 0.156 \mathrm{mmol}$ ) as pale yellow oil in $78 \%$ yield.)

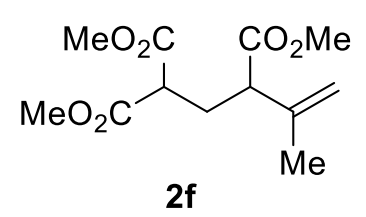

Pale yellow oil; IR (neat): 2955, 1738, 1648, 1437, 1356, 1254, 1159, 1077, 1038, $906 \mathrm{~cm}^{-1} ;{ }^{1} \mathrm{H}$ NMR $\left(500 \mathrm{MHz}, \mathrm{CDCl}_{3}\right) \delta=4.95(\mathrm{~s}, 1 \mathrm{H}), 4.87(\mathrm{~s}, 1 \mathrm{H}), 3.74(\mathrm{~s}, 3 \mathrm{H})$, $3.73(\mathrm{~s}, 3 \mathrm{H}), 3.68(\mathrm{~s}, 3 \mathrm{H}), 3.38(\mathrm{t}, J=7.7 \mathrm{~Hz}, 1 \mathrm{H}), 3.11(\mathrm{t}, J=7.7 \mathrm{~Hz}, 1 \mathrm{H})$, 2.44-2.37 (m, 1H), 2.23-2.16 (m, 1H), $1.72(\mathrm{~s}, 3 \mathrm{H}) ;{ }^{13} \mathrm{C}$ NMR (125 MHz, $\left.\mathrm{CDCl}_{3}\right)$ $\delta=172.9,169.5,169.3,141.1,115.3,52.6,52.1,50.3,49.2,28.6,19.8$ ppm; HRMS (EI) m/z calcd for $\mathrm{C}_{12} \mathrm{H}_{18} \mathrm{O}_{6}[\mathrm{M}]^{+}: 258.1103$. Found: 258.1100 . 
Trimethyl 2-methylpent-4-ene-1,1,3-tricarboxylate (2g)

(1g: $39.6 \mathrm{mg}, 0.20 \mathrm{mmol}$; reaction time: $18 \mathrm{~h}$; reaction temperature: $23{ }^{\circ} \mathrm{C}$; The product was purified by silica gel column chromatography (hexane/AcOEt, 7:1) to afford $\mathbf{2 g}(42.0 \mathrm{mg}, 0.163 \mathrm{mmol}$ ) as colorless oil in $82 \%$ yield; $\mathrm{dr}=1.2 / 1$.)

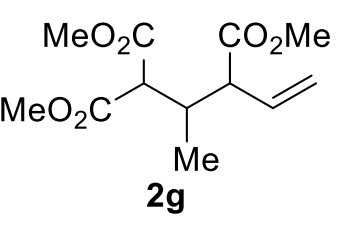

Colorless oil; IR (neat): 2955, 1731, 1638, 1436, 1198, 1162, 1065, 1011, 930, 759 $\mathrm{cm}^{-1} ;{ }^{1} \mathrm{H} \mathrm{NMR}$ (diastereomixture: $500 \mathrm{MHz}, \mathrm{CDCl}_{3}$ ) $\delta=5.84-5.76(\mathrm{~m}$, major $1 \mathrm{H}+$ minor $1 \mathrm{H}), 5.25-5.19(\mathrm{~m}$, major $2 \mathrm{H}+\operatorname{minor} 1 \mathrm{H}), 5.10(\mathrm{~d}, J=17.2 \mathrm{~Hz}$, minor $1 \mathrm{H})$, $3.73(\mathrm{~s}$, minor $3 \mathrm{H}), 3.72(\mathrm{~s}$, major $3 \mathrm{H}+\operatorname{minor} 3 \mathrm{H}), 3.71(\mathrm{~s}$, major $3 \mathrm{H}), 3.69(\mathrm{~s}$, minor $3 \mathrm{H}), 3.68(\mathrm{~s}$, major $3 \mathrm{H}), 3.56(\mathrm{~d}, J=5.7 \mathrm{~Hz}$, major $1 \mathrm{H}), 3.36(\mathrm{~d}, J=8.0 \mathrm{~Hz}$, minor $1 \mathrm{H}), 3.22-3.14$ $(\mathrm{m}$, major $1 \mathrm{H}+$ minor $1 \mathrm{H}), 2.82-2.77(\mathrm{~m}$, minor $1 \mathrm{H}), 2.65-2.60(\mathrm{~m}$, major $1 \mathrm{H}), 1.03(\mathrm{~d}, J=6.9 \mathrm{~Hz}$, major $3 \mathrm{H}), 0.96(\mathrm{~d}, J=6.9 \mathrm{~Hz}$, minor $3 \mathrm{H}) ;{ }^{13} \mathrm{C}$ NMR (diastereomixture: $125 \mathrm{MHz}, \mathrm{CDCl}_{3}$ ) $\delta=173.2,173.0$, 169.2 , 168.7, 168.6, 168.5, 134.2, 132.3, 120.2, 119.8, 54.6, 54.3, 53.2, 52.6, 52.5, 52.44, 52.35, 52.2, 52.0, 51.9, 35.7, 34.8, 14.4, 13.5 ppm; HRMS (EI) m/z calcd for $\mathrm{C}_{12} \mathrm{H}_{18} \mathrm{O}_{6}\left[\mathrm{M}-\mathrm{CH}_{3}\right]^{+}:$243.0869. Found: 243.0863 .

Trimethyl 2,4-dimethylpent-4-ene-1,1,3-tricarboxylate (2h)

(1h: $35.9 \mathrm{mg}, 0.169 \mathrm{mmol}$; reaction time: $18 \mathrm{~h}$; reaction temperature: $23{ }^{\circ} \mathrm{C}$; The product was purified by silica gel column chromatography (hexane/AcOEt, 7:1) to afford $\mathbf{2 h}(31.2 \mathrm{mg}, 0.115 \mathrm{mmol})$ as colorless oil in $68 \%$ yield; $\mathrm{dr}=1.7 / 1$.)

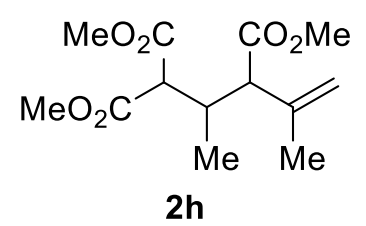

Colorless oil; IR (neat): 2954, 1734, 1645, 1436, 1269, 1224, 1196, 1163, 1013, $904 \mathrm{~cm}^{-1} ;{ }^{1} \mathrm{H}$ NMR (diastereomixture: $\left.500 \mathrm{MHz}, \mathrm{CDCl}_{3}\right) \delta=5.00(\mathrm{~s}$, minor $1 \mathrm{H}$ ), $4.96(\mathrm{~s}$, major $1 \mathrm{H}), 4.95(\mathrm{~s}$, major $1 \mathrm{H}+\operatorname{minor} 1 \mathrm{H}), 3.732(\mathrm{~s}$, major $3 \mathrm{H}+$ minor $3 \mathrm{H}), 3.726(\mathrm{~s}$, major $3 \mathrm{H}), 3.71(\mathrm{~s}$, minor $3 \mathrm{H}), 3.68(\mathrm{~s}$, minor $3 \mathrm{H}), 3.67$ (s, major $3 \mathrm{H}), 3.58(\mathrm{~d}, J=4.0 \mathrm{~Hz}$, major $1 \mathrm{H}), 3.48(\mathrm{~d}, J=4.0 \mathrm{~Hz}$, minor $1 \mathrm{H}), 3.23(\mathrm{~d}, J=10.9 \mathrm{~Hz}$, minor $1 \mathrm{H}), 3.14$ $(\mathrm{d}, J=11.5 \mathrm{~Hz}$, major $1 \mathrm{H}), 2.78-2.71(\mathrm{~m}$, major $1 \mathrm{H}+\operatorname{minor} 1 \mathrm{H}), 1.75(\mathrm{~s}$, minor $3 \mathrm{H}), 1.72$ (s, major $3 \mathrm{H})$, $1.08(\mathrm{~d}, J=6.9 \mathrm{~Hz}$, minor $3 \mathrm{H}), 0.98(\mathrm{~d}, J=6.9 \mathrm{~Hz}$, major $3 \mathrm{H}) ;{ }^{13} \mathrm{C}$ NMR (diastereomixture: $125 \mathrm{MHz}$, $\left.\mathrm{CDCl}_{3}\right) \delta=173.0,172.7,169.6,169.2,168.7,168.5,140.6,140.5,116.8,116.5,56.9,56.6,53.5,52.44$, 52.39, 52.2, 52.1, 52.0, 51.9, 51.8, 33.3, 32.9, 19.9, 19.7, 14.3, 13.7 ppm; HRMS (EI) m/z calcd for $\mathrm{C}_{13} \mathrm{H}_{20} \mathrm{O}_{6}[\mathrm{M}]^{+}: 272.1260$. Found: 272.1258 .

Trimethyl 2-phenylpent-4-ene-1,1,3-tricarboxylate (2i)

(1i: $52.1 \mathrm{mg}, 0.20 \mathrm{mmol}$; reaction time: $18 \mathrm{~h}$; reaction temperature: $23{ }^{\circ} \mathrm{C}$; The product was purified by silica gel column chromatography (hexane/AcOEt, 7:1) to afford $\mathbf{2 i}(51.8 \mathrm{mg}, 0.162 \mathrm{mmol})$ as pale yellow oil in $82 \%$ yield; $\mathrm{dr}=3.2 / 1$ )

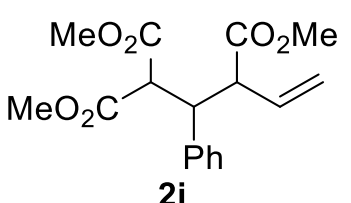

Pale yellow oil; IR (neat): 3031, 2953, 1735, 1496, 1435, 1198, 1019, 930, 751, $703 \mathrm{~cm}^{-1}$; ${ }^{1} \mathrm{H}$ NMR (diastereomixture: $\left.500 \mathrm{MHz}, \mathrm{CDCl}_{3}\right) \delta=7.25-7.11(\mathrm{~m}$, major $5 \mathrm{H}+$ minor $5 \mathrm{H}), 5.81-5.68(\mathrm{~m}$, major $1 \mathrm{H}+\operatorname{minor} 1 \mathrm{H}), 5.28(\mathrm{~d}, J=17.2 \mathrm{~Hz}$, minor $1 \mathrm{H}), 5.22-5.18(\mathrm{~m}$, major $1 \mathrm{H}+$ minor $1 \mathrm{H}), 5.04(\mathrm{~d}, J=17.2 \mathrm{~Hz}$, major $1 \mathrm{H}), 4.07$ $(\mathrm{dd}, J=11.0,6.0 \mathrm{~Hz}$, major $1 \mathrm{H}), 3.95(\mathrm{~d}, J=11.0 \mathrm{~Hz}$, major $1 \mathrm{H}), 3.91-3.87(\mathrm{~m}$, minor $2 \mathrm{H}), 3.77$ (s, major $3 \mathrm{H}), 3.69(\mathrm{~s}$, minor $3 \mathrm{H}), 3.65-3.63(\mathrm{~m}$, minor $1 \mathrm{H}), 3.62(\mathrm{~s}$, major $3 \mathrm{H}), 3.56(\mathrm{dd}, J=11.0,6.0 \mathrm{~Hz}$, major $1 \mathrm{H}), 3.43$ (s, minor $3 \mathrm{H}), 3.412$ (s, minor $3 \mathrm{H}$ ), 3.405 (s, major $3 \mathrm{H}) ;{ }^{13} \mathrm{C}$ NMR (diastereomixture: $125 \mathrm{MHz}$, 
$\left.\mathrm{CDCl}_{3}\right) \delta=172.3,172.0,168.5,168.3,167.9,167.8,137.9,136.7,134.0,132.3,128.9,128.8,128.2$, 128.1, 127.5, 120.0, 119.7, 55.31, 55.29, 54.8, 52.8, 52.5, 52.4, 52.3, 51.9, 51.7, 47.1, 46.3 ppm; HRMS (ESI) $\mathrm{m} / \mathrm{z}$ calcd for $\mathrm{C}_{17} \mathrm{H}_{20} \mathrm{O}_{6}[\mathrm{M}+\mathrm{Na}]^{+}: 343.1158$. Found: 343.1148 .

\section{(D) Determination of Relative Configuration}

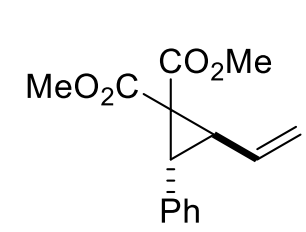

$1 \mathrm{i}$

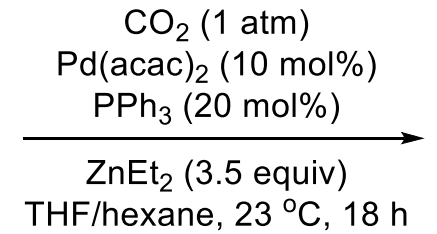

THF/hexane, $23^{\circ} \mathrm{C}, 18 \mathrm{~h}$

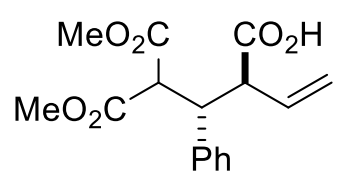

2i': $87 \%$

$\mathrm{dr}=4.2: 1$

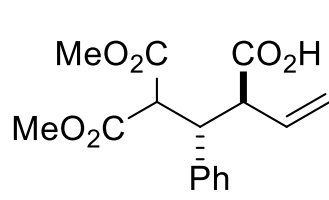

$\mathrm{dr}=4.2: 1$

1) $\mathrm{Pd} / \mathrm{C}(10 \mathrm{~mol} \%), \mathrm{H}_{2}$ (1 atm), MeOH, rt, $5 \mathrm{~h}$

2) $\mathrm{ClCO}_{2} i-\mathrm{Bu}$ (1.0 equiv), $\mathrm{Et}_{3} \mathrm{~N}$ (1.1 equiv), THF, $-20^{\circ} \mathrm{C}, 1 \mathrm{~h}$

3) $\mathrm{NaBH}_{4}$ (3.0 equiv), $\mathrm{THF} / \mathrm{H}_{2} \mathrm{O},-20^{\circ} \mathrm{C}$ to $\mathrm{rt}, 1 \mathrm{~h}$

4) $\mathrm{Zn}_{4}\left(\mathrm{OCOCF}_{3}\right)_{6}(10 \mathrm{~mol} \%)$, xylene, $160^{\circ} \mathrm{C}$, sealed, $24 \mathrm{~h}$

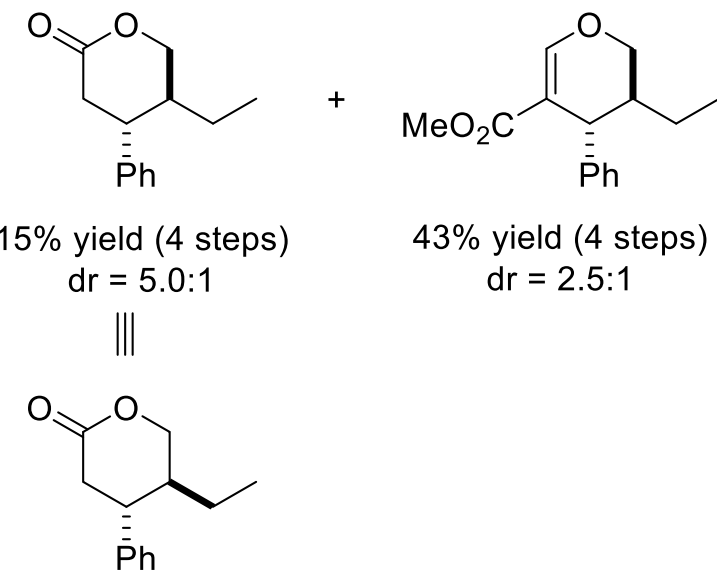

$\mathrm{Pd}(\mathrm{acac})_{2}(34.3 \mathrm{mg}, 0.112 \mathrm{mmol}, 10 \mathrm{~mol} \%)$ and $\mathrm{PPh}_{3}(58.8 \mathrm{mg}, 0.224 \mathrm{mmol}, 20 \mathrm{~mol} \%)$ were placed on the bottom of a recovery flask containing a stirring bar. The flask was evacuated and refilled with $\mathrm{CO}_{2}$ (balloon) five times. After the addition of dry THF $(5 \mathrm{~mL})$, the mixture was stirred at room temperature for $10 \mathrm{~min}$ under $\mathrm{CO}_{2}$ atmosphere (1 atm: balloon). Vinylcyclopropane 1i (290.4 mg, $\left.1.12 \mathrm{mmol}\right)$ and $\mathrm{ZnEt}_{2}\left(1 \mathrm{M}\right.$ solution in hexane, $3.92 \mathrm{~mL}, 3.92 \mathrm{mmol}, 3.5$ equiv) were added at $0{ }^{\circ} \mathrm{C}$. The resulting reaction mixture was stirred at $23{ }^{\circ} \mathrm{C}$ for $18 \mathrm{~h}$. After cooling to $0{ }^{\circ} \mathrm{C}, \mathrm{HCl}$ aq. $(3 \mathrm{M})$ and water were added, and the mixture $(\mathrm{pH}<2)$ was extracted with AcOEt three times. The combined organic layer was washed with brine and dried over $\mathrm{Na}_{2} \mathrm{SO}_{4}$. After the solvent was removed under reduced pressure, and the residue was purified by silica gel column chromatography (hexane/AcOEt, 4:1 2:3), affording 5-methoxy-4-(methoxycarbonyl)-5-oxo-3-phenyl-2-vinylpentanoic acid (2i') (297.3 $\mathrm{mg}, 0.971 \mathrm{mmol})$ as amorphous in $87 \%$ yield with $4: 2: 1 \mathrm{dr}$.

5-Methoxy-4-(methoxycarbonyl)-5-oxo-3-phenyl-2-vinylpentanoic acid (2i')

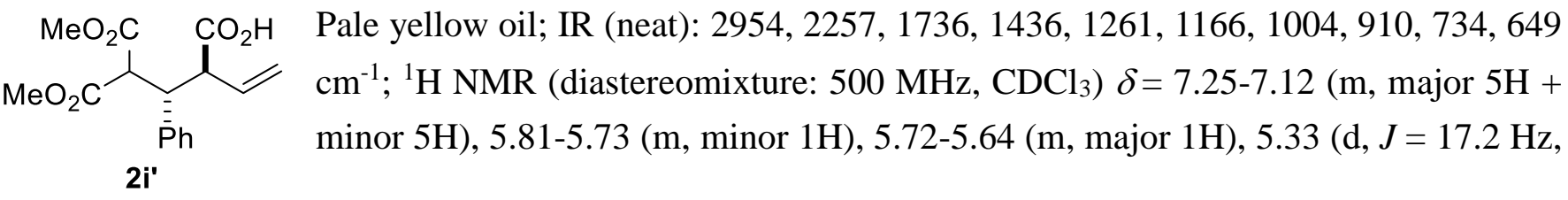


minor $1 \mathrm{H}), 5.26-5.22(\mathrm{~m}$, major $1 \mathrm{H}+\operatorname{minor} 1 \mathrm{H}), 5.08(\mathrm{~d}, J=17.2 \mathrm{~Hz}$, major $1 \mathrm{H}), 4.07(\mathrm{dd}, J=11.4,5.6$ $\mathrm{Hz}$, major $1 \mathrm{H}), 3.94(\mathrm{~d}, J=11.4 \mathrm{~Hz}$, major $1 \mathrm{H}), 3.93-3.85(\mathrm{~m}$, minor $2 \mathrm{H}), 3.75$ (s, major $3 \mathrm{H}), 3.68(\mathrm{~s}$, minor $3 \mathrm{H}), 3.66-3.64(\mathrm{~m}$, minor $1 \mathrm{H}), 3.59(\mathrm{dd}, J=9.7,5.6 \mathrm{~Hz}$, major $1 \mathrm{H}), 3.42(\mathrm{~s}$, minor $3 \mathrm{H}), 3.41(\mathrm{~s}$, major $3 \mathrm{H}$ ); ${ }^{13} \mathrm{C}$ NMR (diastereomixture: $125 \mathrm{MHz}, \mathrm{CDCl}_{3}$ ) $\delta=177.6,177.1,168.3,167.7,136.4,133.4$, 131.7, 129.1, 128.8, 128.3, 128.2, 127.7, 127.6, 120.4, 55.2, 54.8, 54.7, 52.8, 52.5, 52.4, 52.3, 52.1, 46.8, 46.0 ppm; HRMS (ESI) m/z calcd for $\mathrm{C}_{16} \mathrm{H}_{18} \mathrm{O}_{6}[\mathrm{M}+\mathrm{Na}]^{+}: 329.1001$. Found: 329.0998 .

2i' $(38.0 \mathrm{mg}, 0.124 \mathrm{mmol}, 1$ equiv) and $5 \% \mathrm{Pd} / \mathrm{C}(52.8 \mathrm{mg}, 0.025 \mathrm{mmol}, 20 \mathrm{~mol} \%)$ were placed on the bottom of a recovery flask containing a stirring bar. The flask was evacuated and refilled with $\mathrm{H}_{2}$ (balloon) five times. After the addition of $\mathrm{MeOH}(6 \mathrm{~mL})$, the mixture was stirred at room temperature for $5 \mathrm{~h}$ under $\mathrm{H}_{2}$ atmosphere (1 atm: balloon). The reaction mixture was passed through a short pad of Celite ${ }^{\circledR}$. The crude mixture was used for next reaction without further purification.

The crude mixture was placed on the bottom of a recovery flask containing a stirring bar. THF ( $1 \mathrm{~mL})$ was added and the solution was cooled to $-20^{\circ} \mathrm{C}$. $\mathrm{Et}_{3} \mathrm{~N}(19.0 \mu \mathrm{L}, 0.136 \mathrm{mmol}, 1.1$ equiv) in THF (95.1 $\mu \mathrm{L})$ and $\mathrm{ClCO}_{2} i$-Bu $(16.1 \mu \mathrm{L}, 0.124 \mathrm{mmol}, 1.0$ equiv) in THF $(80.5 \mu \mathrm{L})$ were then added dropwise and the mixture was stirred at $-20^{\circ} \mathrm{C}$. After $1 \mathrm{~h}, \mathrm{NaBH}_{4}\left(14.1 \mathrm{mg}, 0,372 \mathrm{mmol}, 3.0\right.$ equiv) in $\mathrm{H}_{2} \mathrm{O}(1 \mathrm{~mL})$ was added and the resulting reaction mixture was stirred at $-20{ }^{\circ} \mathrm{C}$ to room temperature for $1 \mathrm{~h}$. Then the mixture was extracted with AcOEt three times. The combined organic layer was washed with brine and dried over $\mathrm{Na}_{2} \mathrm{SO}_{4}$. After the solvent was removed under reduced pressure, and the obtained crude mixture was used for next reaction without further purification.

The crude mixture and $\mathrm{Zn}_{4}\left(\mathrm{OCOCF}_{3}\right)_{6}$ were placed on the bottom of a sealed tube containing a stirring bar. After xylene $(1 \mathrm{~mL})$ was added to the tube, the system was closed and stirred at $160{ }^{\circ} \mathrm{C}$ for $24 \mathrm{~h} .{ }^{6}$ The reaction mixture was passed through a short pad of Celite ${ }^{\circledR}$. After the solvent was removed under reduced pressure, and the residue was purified by preparative thin-layer-chromatography (hexane/AcOEt, 4:1), affording 5-ethyl-4-phenyltetrahydro-2H-pyran-2-one $(3.9 \mathrm{mg}, 0.019 \mathrm{mmol})$ as colorless oil in $15 \%$ yield with 5.0:1 dr in 4 steps. The spectral data was completely matched with the reported values. ${ }^{7}$

5-ethyl-4-phenyltetrahydro-2H-pyran-2-one (diastereomixture)

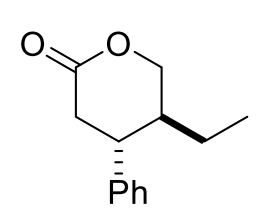

Colorless oil; ${ }^{1} \mathrm{H}$ NMR (diastereomixture: $\left.500 \mathrm{MHz}, \mathrm{CDCl}_{3}\right) \delta=7.37-7.26(\mathrm{~m}$, major $3 \mathrm{H}$ + minor $3 \mathrm{H}), 7.18(\mathrm{~d}, J=8.0 \mathrm{~Hz}$, major $2 \mathrm{H}), 7.14(\mathrm{~d}, J=8.0 \mathrm{~Hz}$, minor $2 \mathrm{H}), 4.50(\mathrm{dd}, J$ $=11.2,4.6 \mathrm{~Hz}$, major $1 \mathrm{H}), 4.35(\mathrm{dd}, J=11.5,4.0 \mathrm{~Hz}$, minor $1 \mathrm{H}), 4.18(\mathrm{dd}, J=11.5,8.6$ $\mathrm{Hz}$, minor $1 \mathrm{H}), 4.10(\mathrm{dd}, J=11.2,9.7 \mathrm{~Hz}$, major $1 \mathrm{H}), 3.42-3.38(\mathrm{~m}$, minor $1 \mathrm{H})$, 2.90-2.83 (m, major $2 \mathrm{H}+$ minor $2 \mathrm{H}), 2.68-2.61(\mathrm{~m}$, major $1 \mathrm{H}), 2.12-1.98(\mathrm{~m}$, major $1 \mathrm{H}+$ minor $1 \mathrm{H})$, 1.48-1.39 (m, major $1 \mathrm{H}+$ minor $1 \mathrm{H}), 1.23-1.05$ (major $1 \mathrm{H}+\operatorname{minor} 1 \mathrm{H}), 0.94(\mathrm{t}, J=7.4 \mathrm{~Hz}$, minor $3 \mathrm{H})$, $0.84(\mathrm{t}, J=7.4 \mathrm{~Hz}$, major $3 \mathrm{H}) \mathrm{ppm}$

${ }^{6}$ Hirata, G.; Onodera, G.; Kimura, M. Synlett 2014, 25, 2306.

7 Landa, A.; Maestro, M.; Masdeu, C.; Puente, Á.; Vera, S.; Oiarbide, M.; Palomo, C. Chem. Eur. J. 2009, $15,1562$. 
trans-5-ethyl-4-phenyltetrahydro-2H-pyran-2-one (major diastereomer)

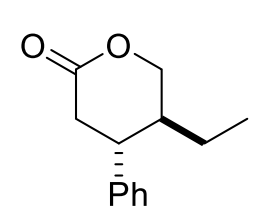
Colorless oil; IR (neat): 2963, 2924, 1739, 1455, 1405, 1225, 1110, 1058, 757, $702 \mathrm{~cm}^{-1}$; ${ }^{1} \mathrm{H}$ NMR $\left(500 \mathrm{MHz}, \mathrm{CDCl}_{3}\right) \delta=7.35(\mathrm{t}, J=7.4 \mathrm{~Hz}, 2 \mathrm{H}), 7.29-7.27(\mathrm{~m}, 1 \mathrm{H}), 7.18(\mathrm{~d}, J=$ $7.4 \mathrm{~Hz}, 2 \mathrm{H}), 4.50(\mathrm{dd}, J=11.5,4.6 \mathrm{~Hz}, 1 \mathrm{H}), 4.09(\mathrm{dd}, J=11.5,9.2 \mathrm{~Hz}, 1 \mathrm{H}), 2.90-2.83$ $(\mathrm{m}, 2 \mathrm{H}), 2.68-2.61(\mathrm{~m}, 1 \mathrm{H}), 2.06-1.98(\mathrm{~m}, 1 \mathrm{H}), 1.48-1.39(\mathrm{~m}, 1 \mathrm{H}), 1.24-1.15(\mathrm{~m}, 1 \mathrm{H})$, $0.84(\mathrm{t}, J=7.4 \mathrm{~Hz}, 3 \mathrm{H}) ;{ }^{1} \mathrm{H}$ NMR $\left(500 \mathrm{MHz}, \mathrm{C}_{6} \mathrm{D}_{6}\right) \delta=7.09-7.05(\mathrm{~m}, 2 \mathrm{H}), 7.04-7.00(\mathrm{~m}, 1 \mathrm{H}), 6.76-6.74$ $(\mathrm{m}, 2 \mathrm{H}), 3.88(\mathrm{dd}, J=11.3,4.6 \mathrm{~Hz}, 1 \mathrm{H}), 3.38(\mathrm{dd}, J=11.3,9.5 \mathrm{~Hz}, 1 \mathrm{H}), 2.43(\mathrm{dd}, J=16.9,6.6 \mathrm{~Hz}, 1 \mathrm{H})$, $2.22(\mathrm{dd}, J=16.9,10.0 \mathrm{~Hz}, 1 \mathrm{H}), 2.10-2.04(\mathrm{~m}, 1 \mathrm{H}), 1.39-1.31(\mathrm{~m}, 1 \mathrm{H}), 0.98-0.89(\mathrm{~m}, 1 \mathrm{H}), 0.65-0.55(\mathrm{~m}$, $1 \mathrm{H}), 0.36(\mathrm{t}, J=7.4 \mathrm{~Hz}, 3 \mathrm{H}) ;{ }^{13} \mathrm{C} \mathrm{NMR}\left(125 \mathrm{MHz}, \mathrm{CDCl}_{3}\right) \delta=171.4,142.6,129.0,128.6,127.2,72.0$, 43.7, 41.1, 37.3, 23.2, 11.1 ppm; HRMS (EI) m/z calcd for $\mathrm{C}_{13} \mathrm{H}_{16} \mathrm{O}_{2}[\mathrm{M}]^{+}:$204.1150. Found: 204.1144.

NOE experiment was conducted in $\mathrm{C}_{6} \mathrm{D}_{6}$ to confirm its relative configuration as 4,5-trans.

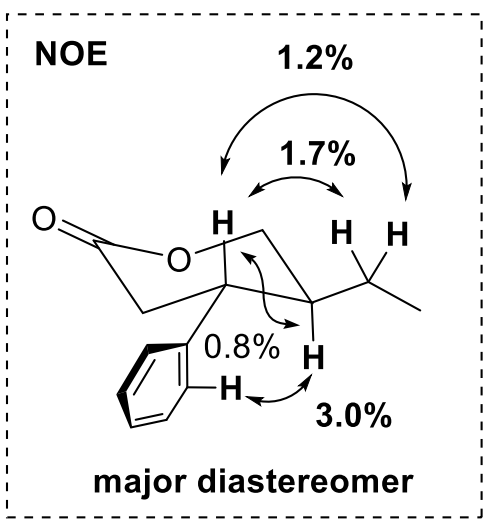




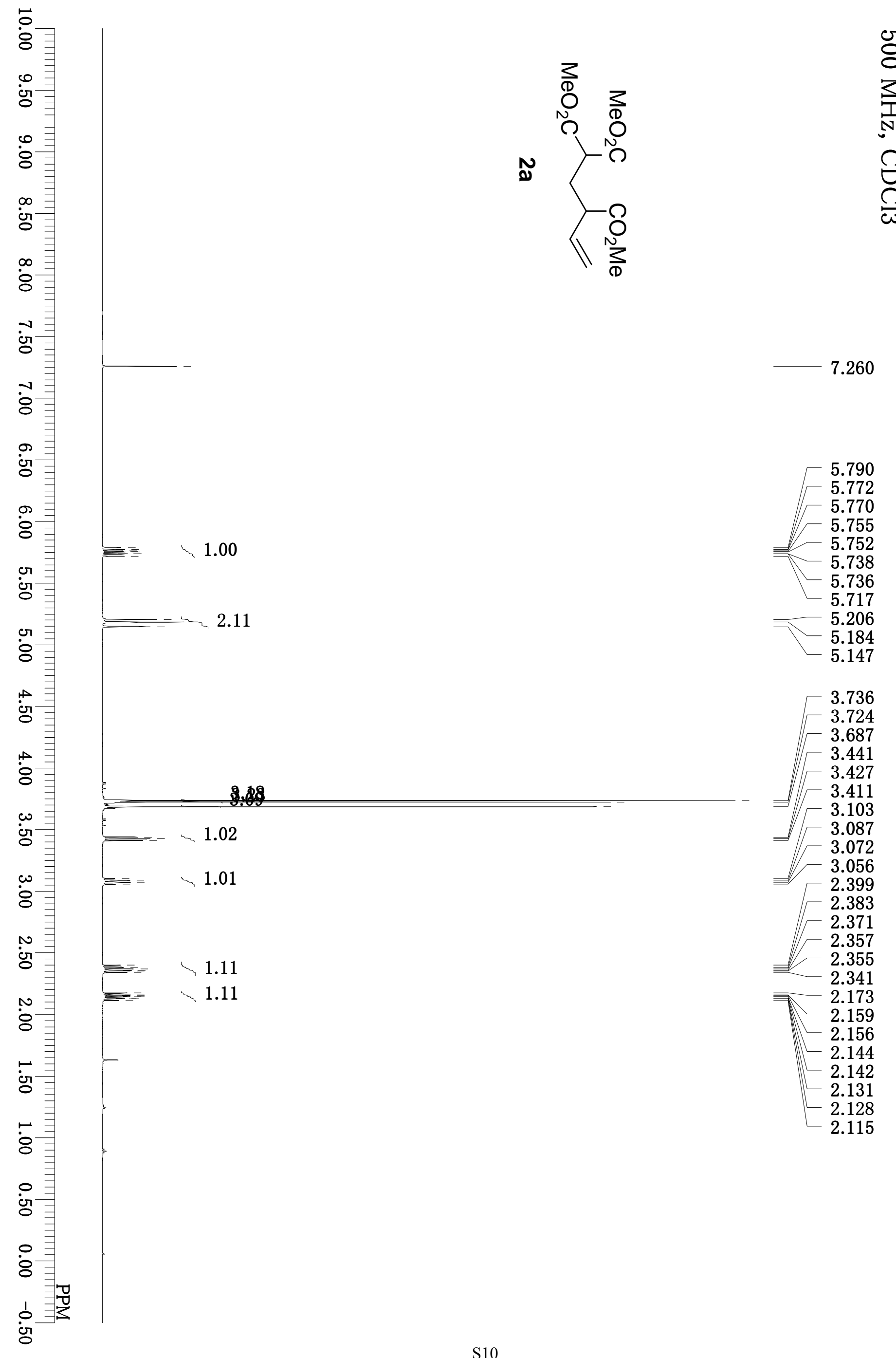




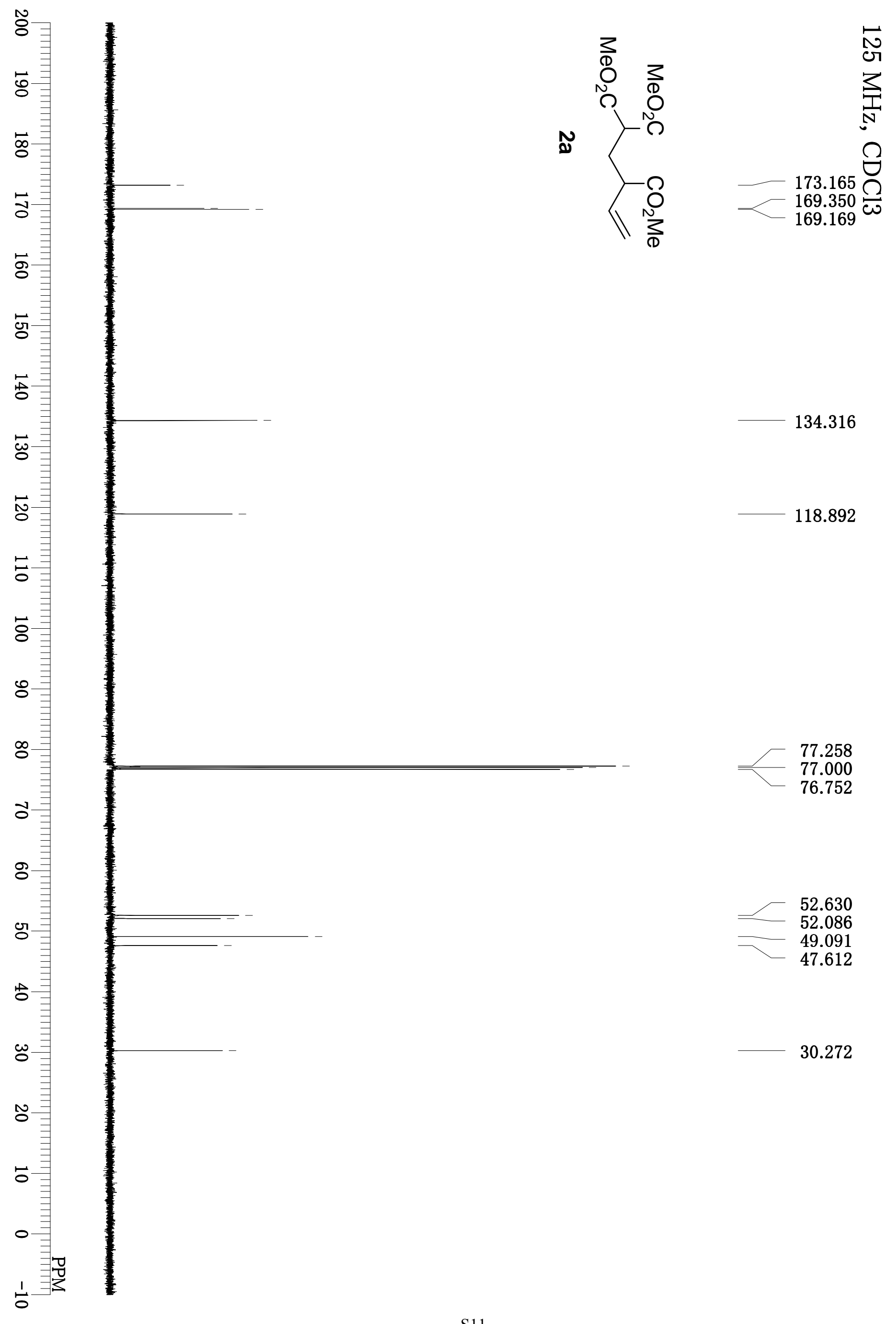




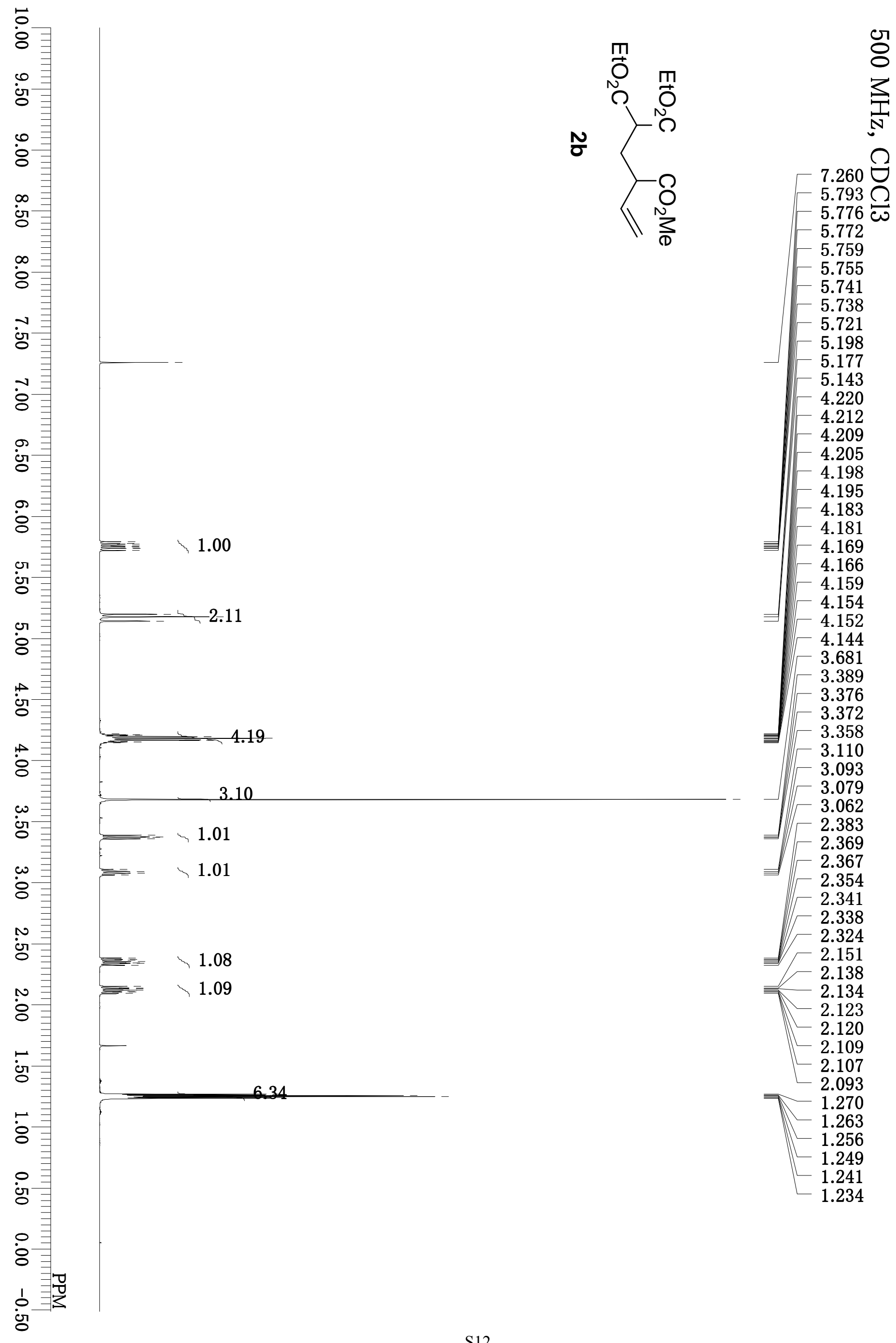




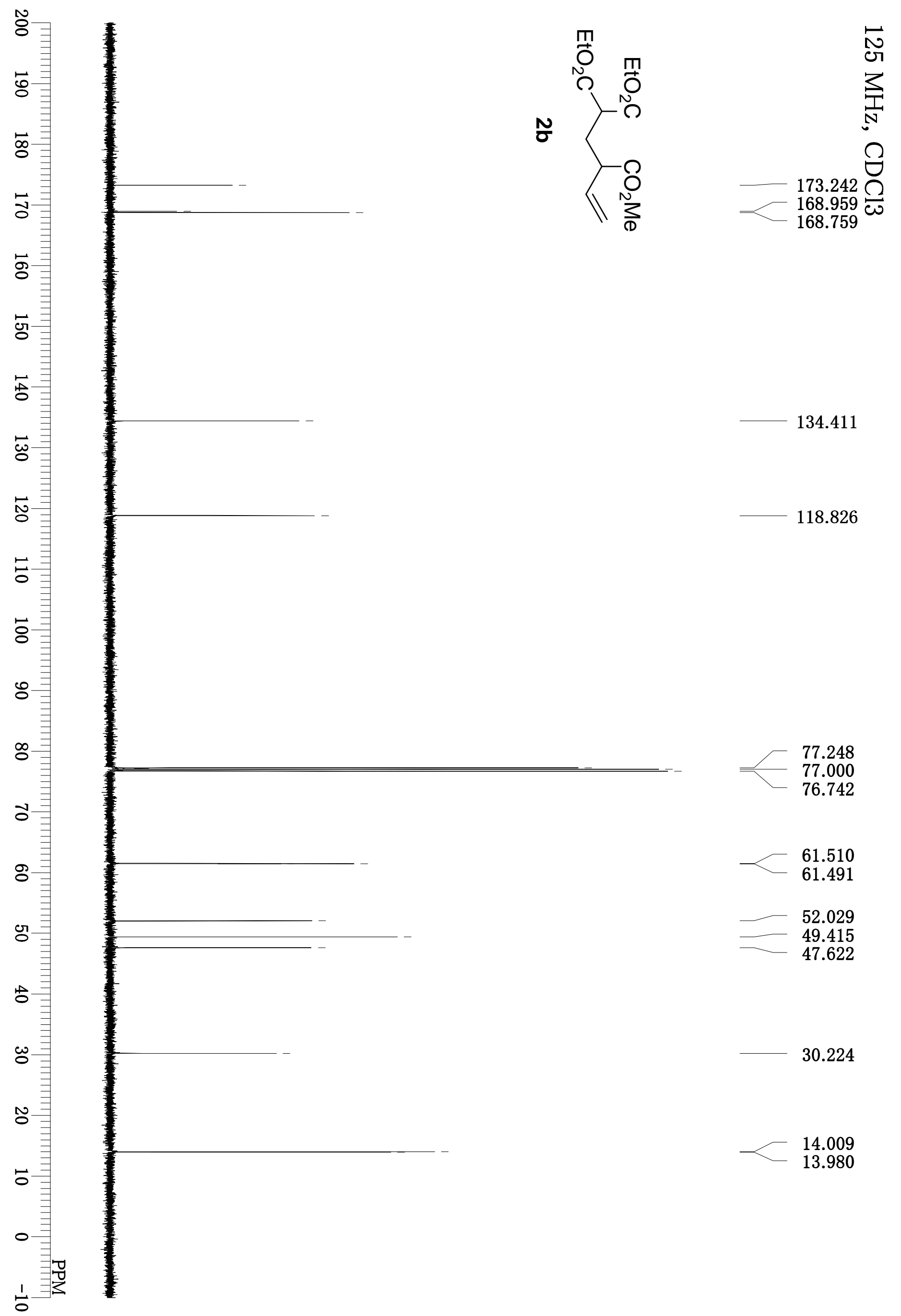




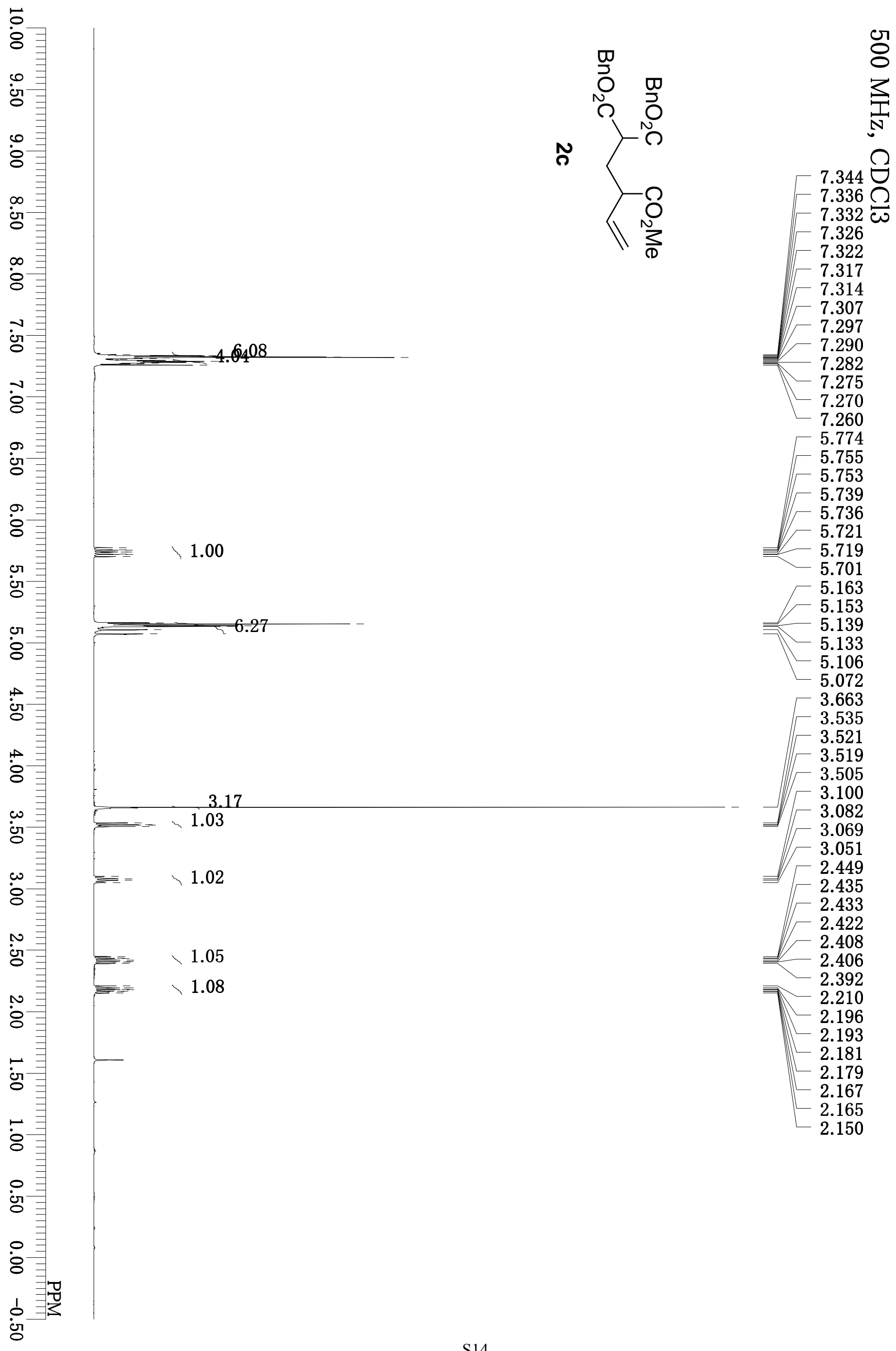




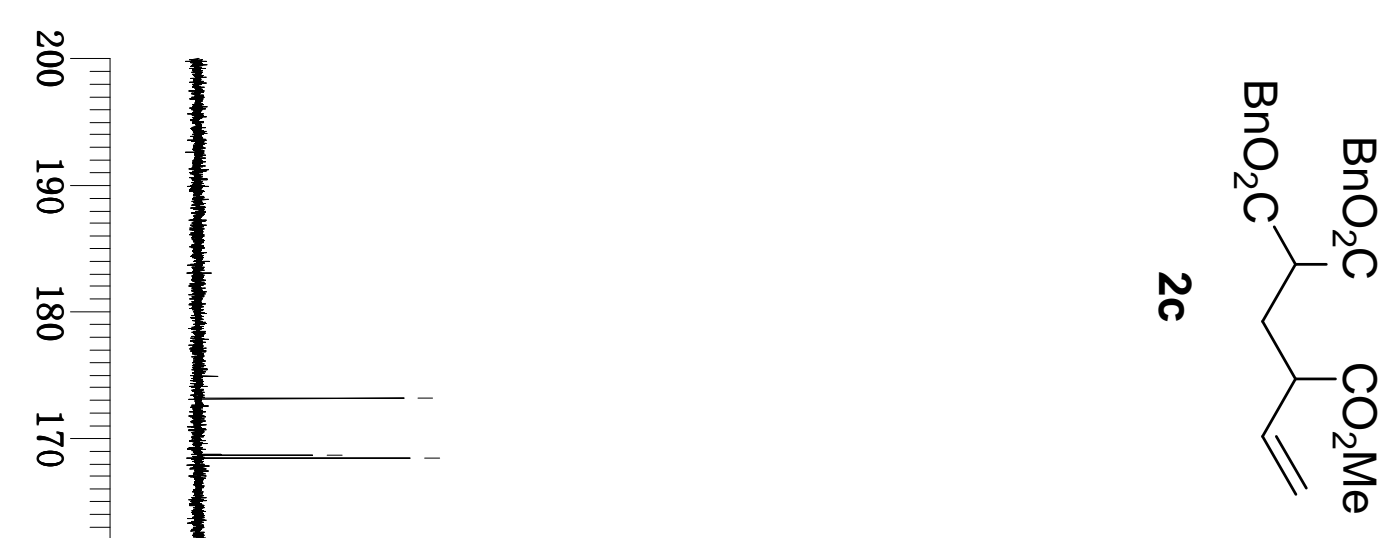

135.184

135.146

134.249

128.516

๘્

128.345

128.164

ज

118.950

。

용

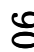

$\infty$

구

옹

o

足

.

今

홍

。

$$
\stackrel{\square}{\circ}
$$$$
\stackrel{1}{0}
$$

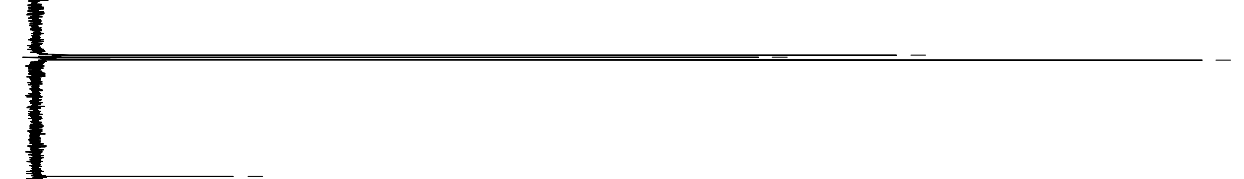




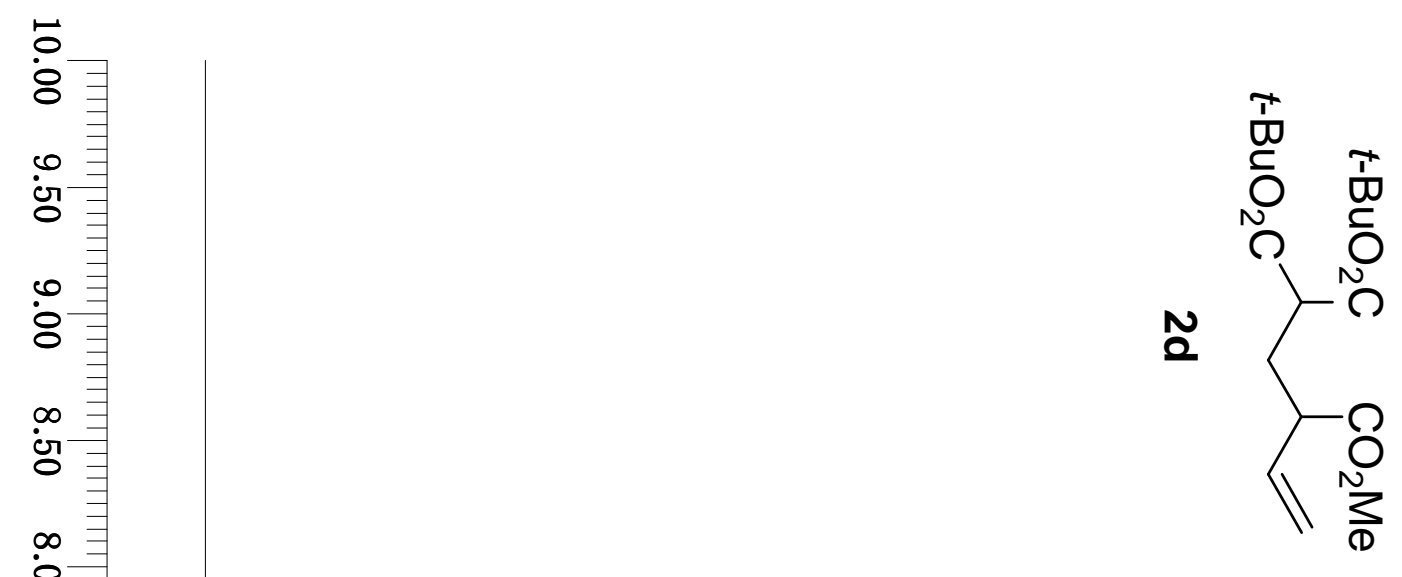

$\ddot{8}$

잉

$\backslash 1.00$

5.807

5.790

5.786

$\stackrel{8}{8}$

5.772

5.769

5.755

5.753

5.735

2.06

5.196

5.178

5.146

$-3.690$

3.196

3.182

$-3.180$

3.166

3.113

3.096

3.082

3.065

2.300

1:80

2.286

2.283

2.272

2.258

2.256

2.242

2.059

2.045

2.041

2.031

2.028

2.017

2.014

2.000

1.455

1.447 


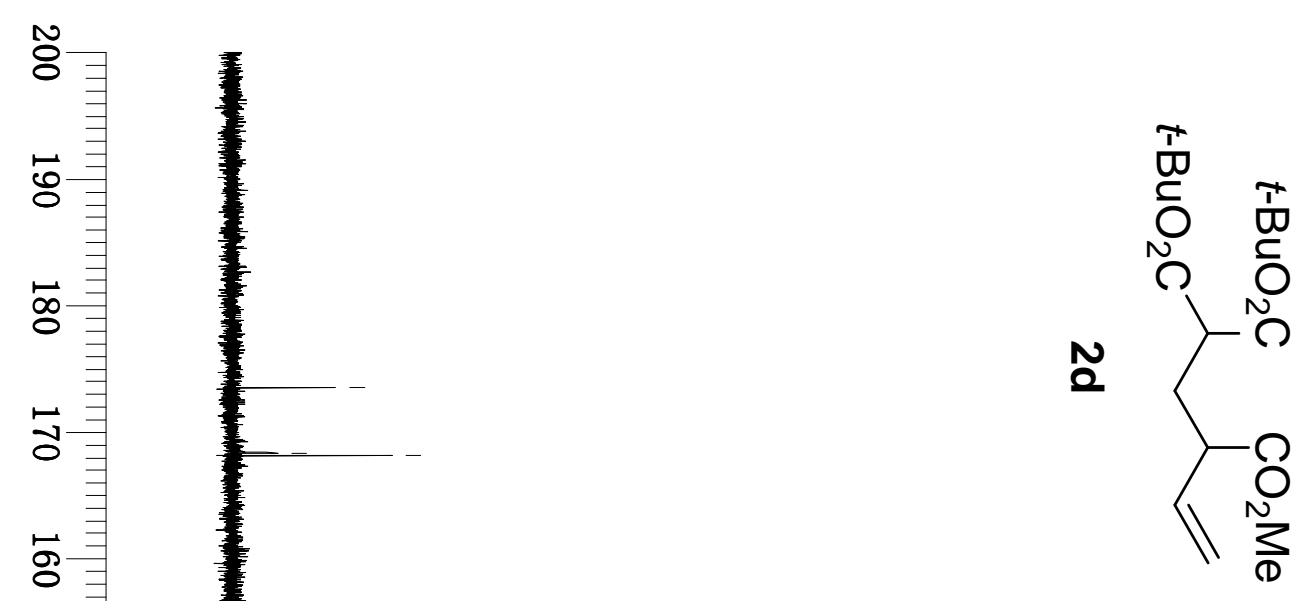

5

每

घ.

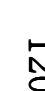
173.518 168.377

ิㅜㅇ

。

○.

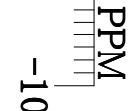

\section{(}




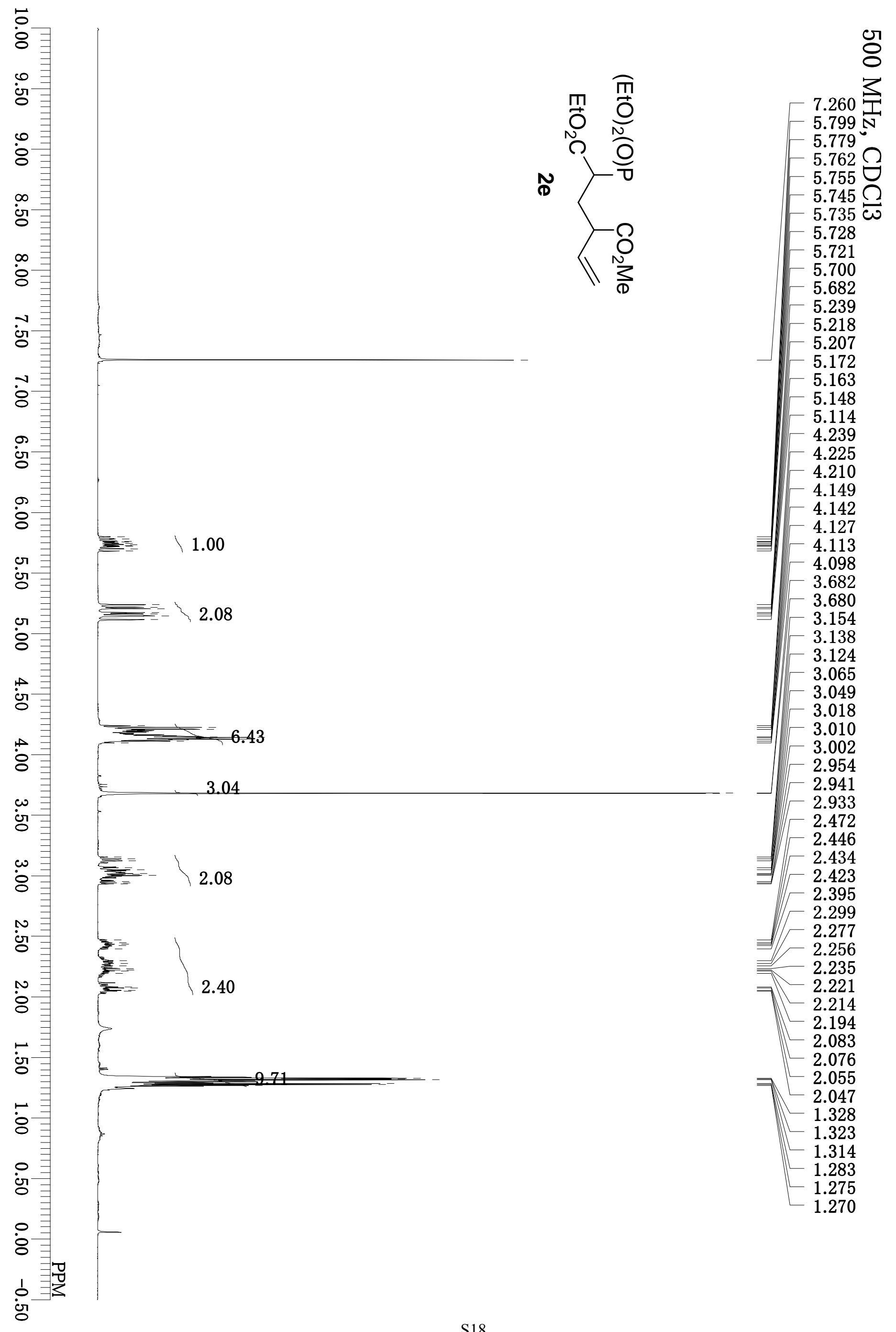




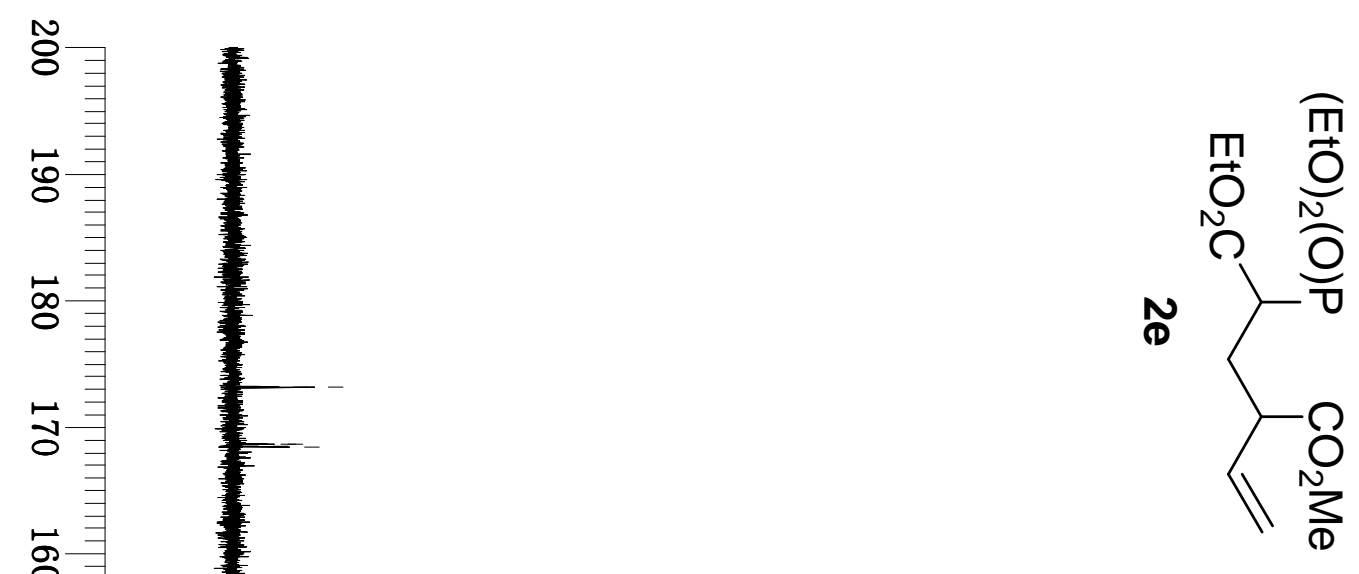

苦 e.

ง

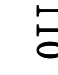$$
8
$$

$$
\varnothing
$$$$
\text { \& }
$$

$$
\widetilde{0}
$$

$$
8
$$
o

$$
\text { to }
$$$$
\text { क. }
$$$$
\text { a }
$$

$$
\begin{aligned}
& 5 \\
& \circ \\
& \vdots
\end{aligned}
$$




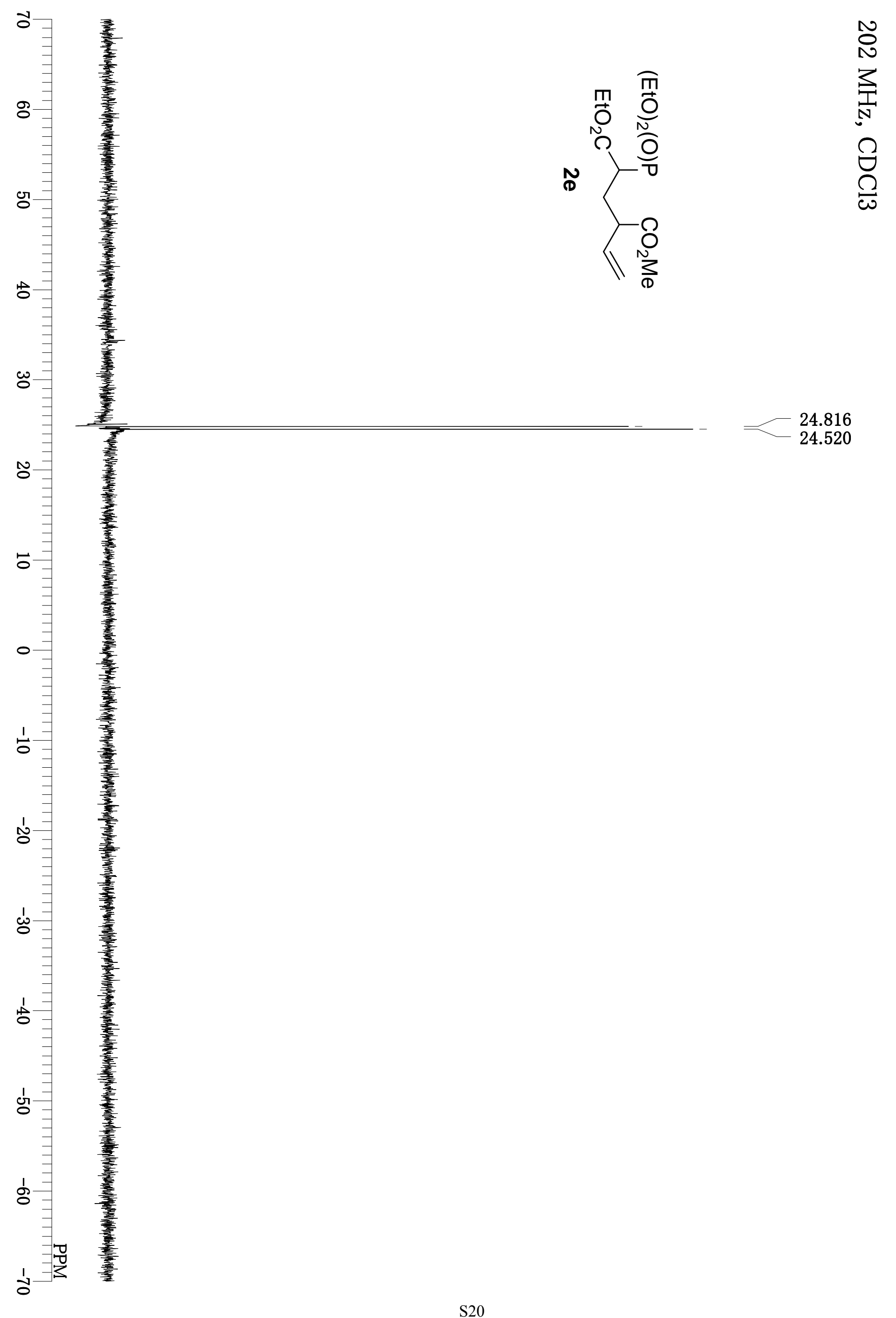




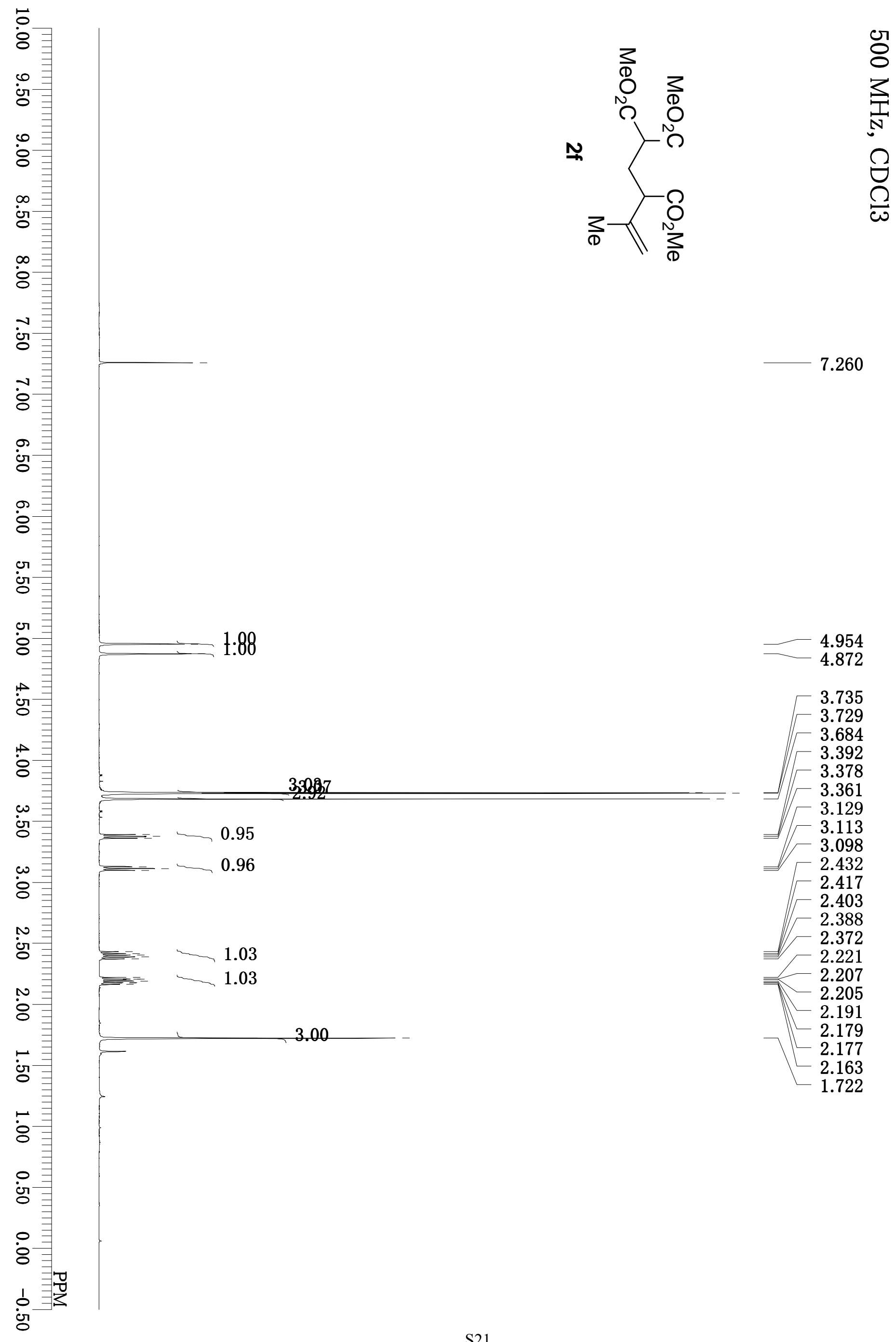



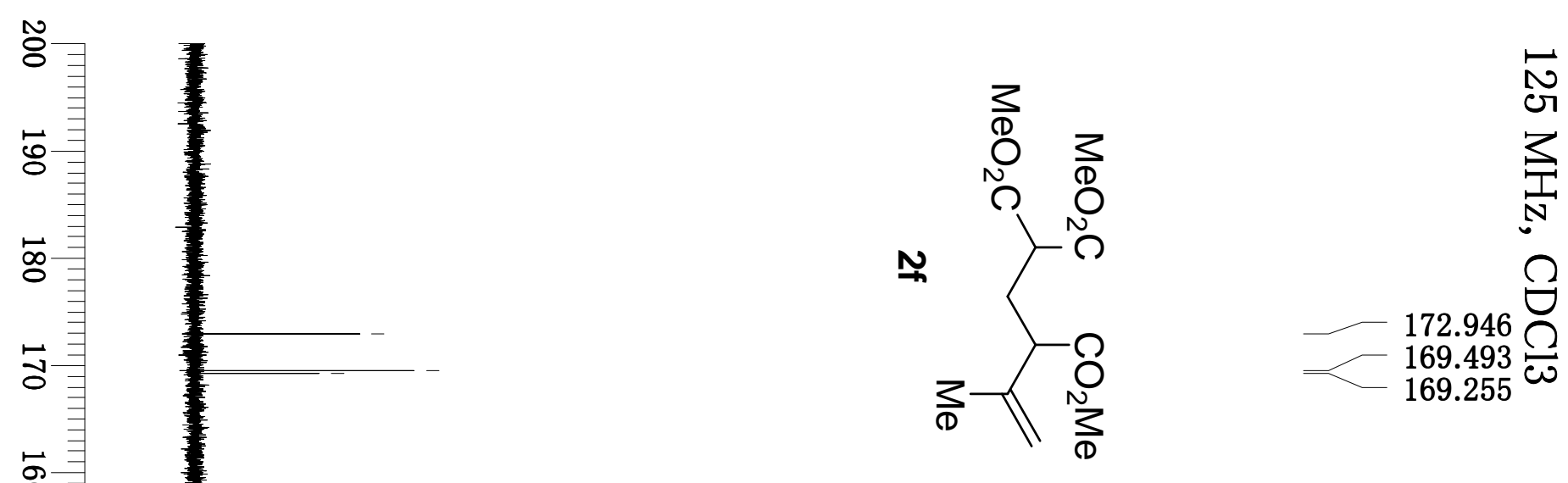

ह

告

e્o

ิㅗㅇ

ま

홍

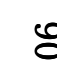

$\infty$

o

$$
\text { : }
$$

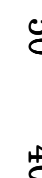$$
\text { t }
$$$$
\text { w }
$$

$\approx$$$
5
$$$$
\text { 。 }
$$$$
\text { 告謨 }
$$ 


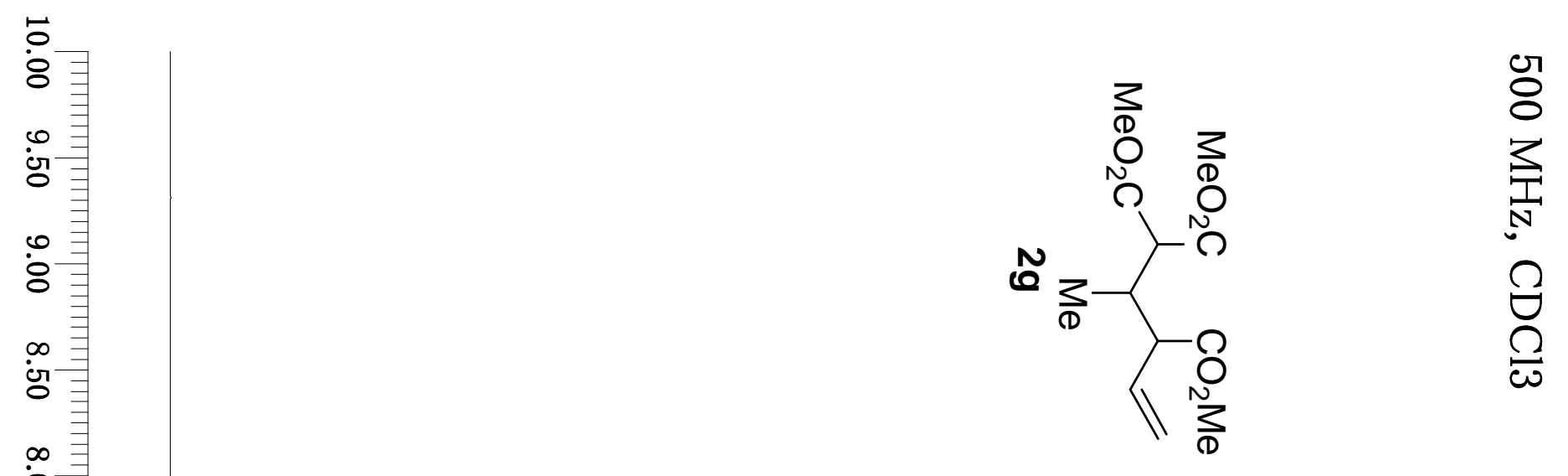

$\dot{8}$

$-5.120$

5.085 3.734

o

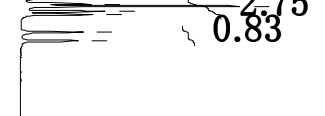

3.721
-3.714

3.714 3.692

3.684

3.561

3.550

3.371

3.355

3.212

3.199

级 1

3.192

3.180

3.172

3.153

3.135

2.816

2.802

0.81

2.786

2.772

2.644

2.630

2.627

2.619

2.614

2.600

1.036

1.022

0.967

0.953

ن்

웅

ن 


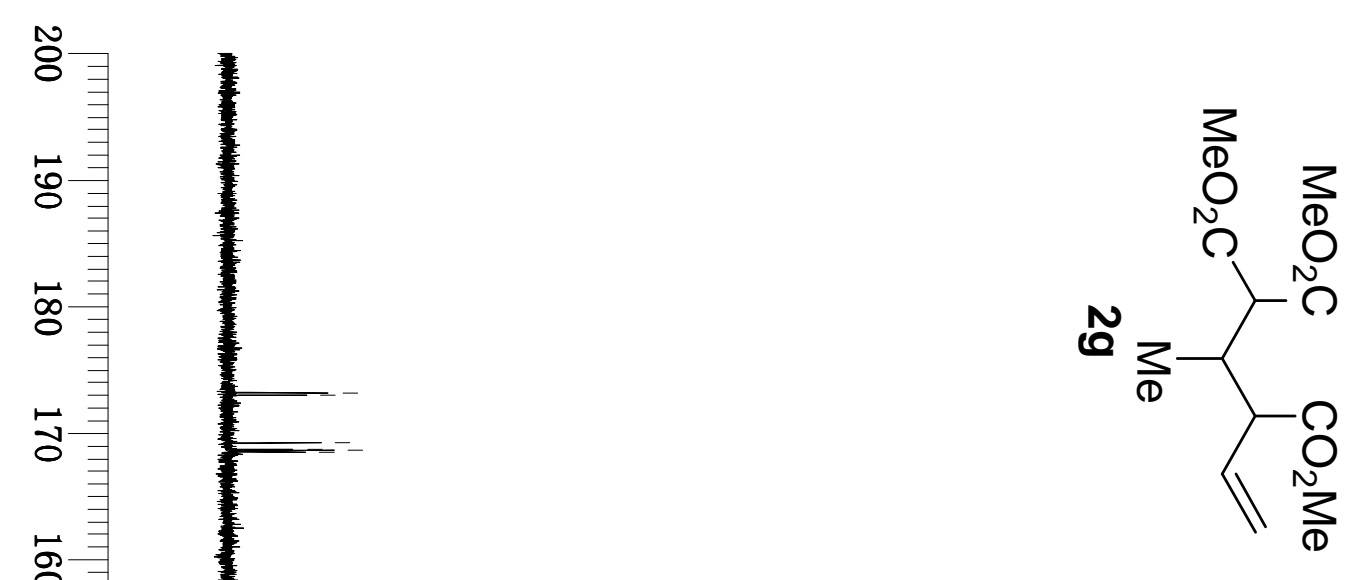

ิㅗㅇ o

s

8 。

$$
\text { वे }
$$
g$$
\text { to }
$$$$
\text { क }
$$

$\approx$

$$
5
$$

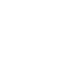

$$
\text { ○ }
$$

㟔 $173.194 \Omega$ 172.994 $169.236 \Omega$ 168.740 ம

168.616

168.530

120.151

119.789

77.258

77.000

76.742

54.642

54.251

53.202

52.620

52.515

52.439

52.353

52.200

51.981

51.857

35.651

34.774

14.448

13.465 


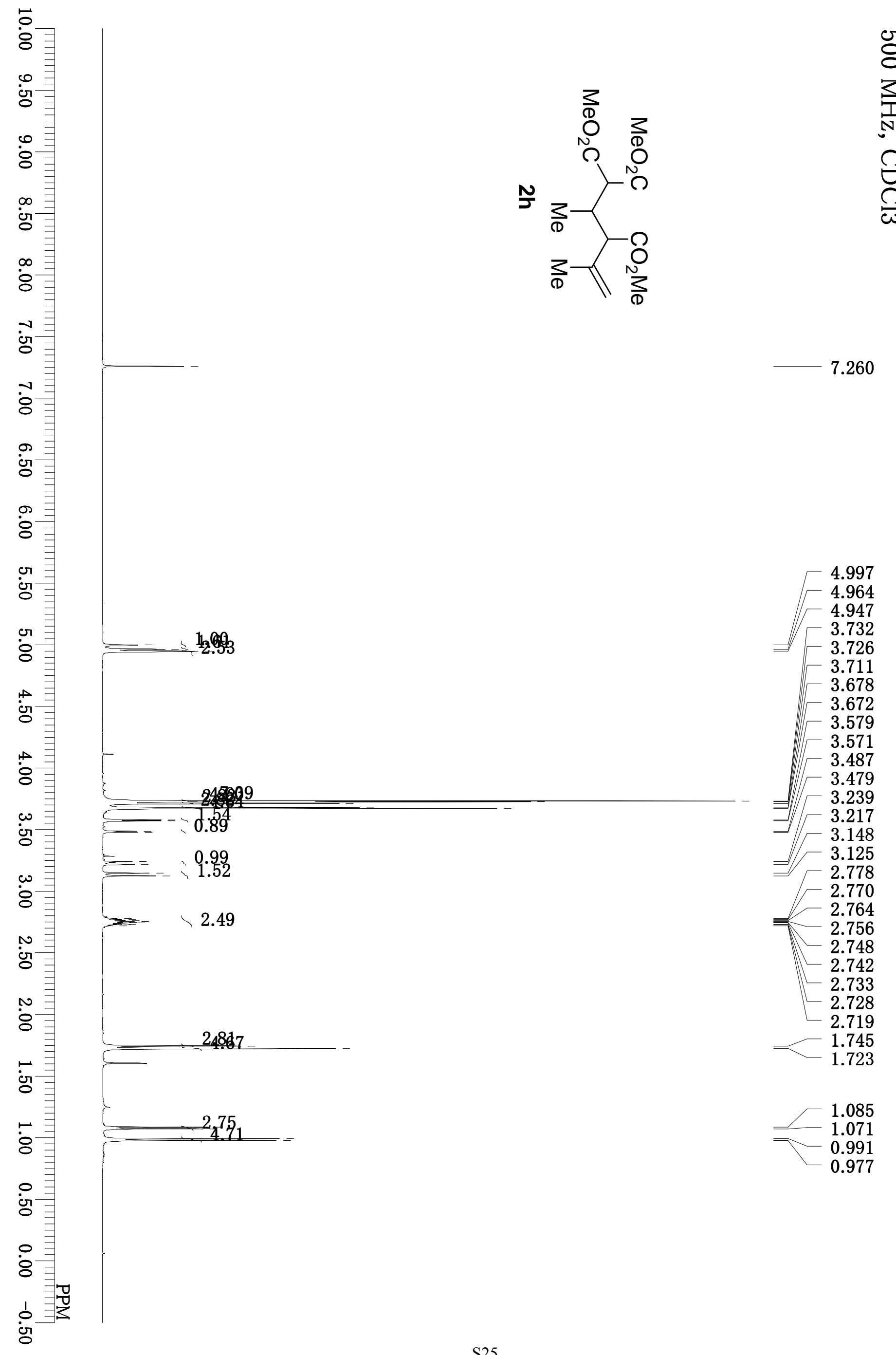




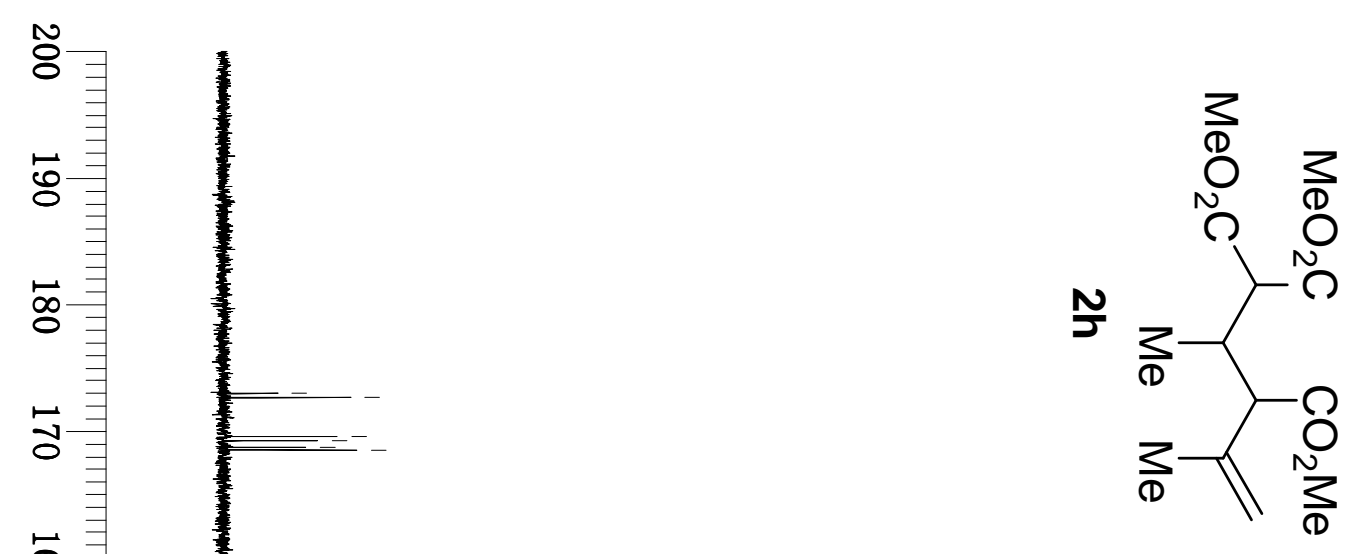

。

응

8

$\stackrel{\infty}{\circ}$

구

。

c

年

.

ช

$\vec{\circ}$

。

占寻

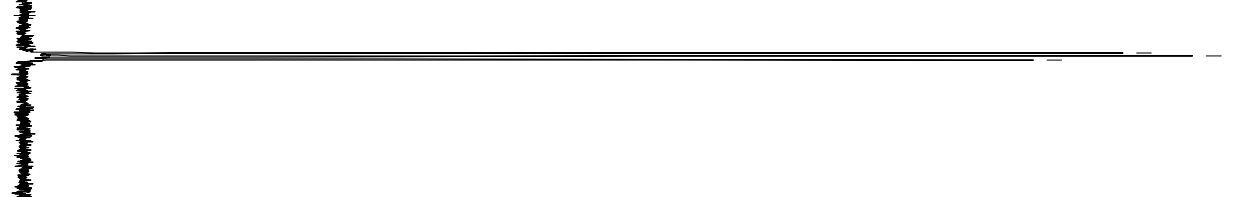

77.258

77.000

76.742

56.874

56.569

53.459

52.439

52.391

52.210

52.086

52.038

51.933

51.847

33.314

32.933

19.885

19.722

14.295

13.704 


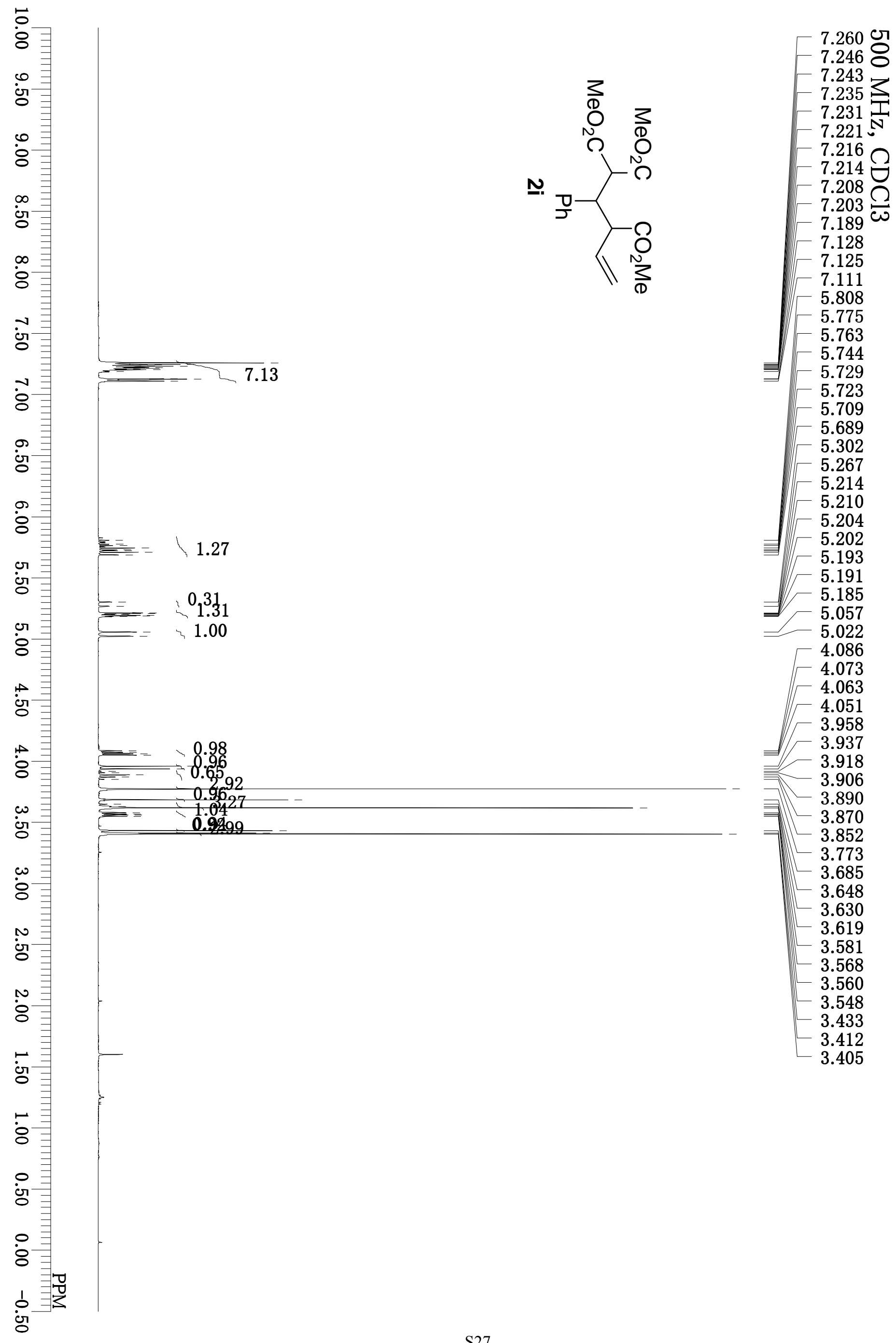




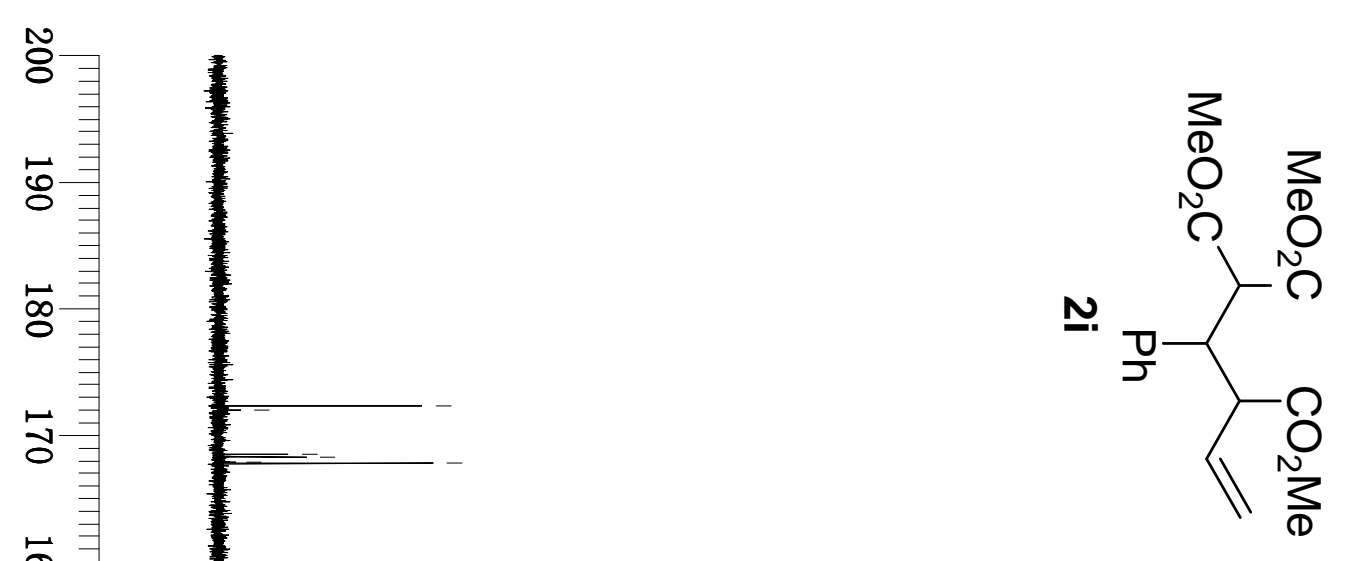



\&

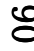

$\infty$

77.248

77.000

76.742

ò

55.310

55.291

54.833

52.773

52.487

52.353

52.267

51.886

51.733

47.107

46.287 


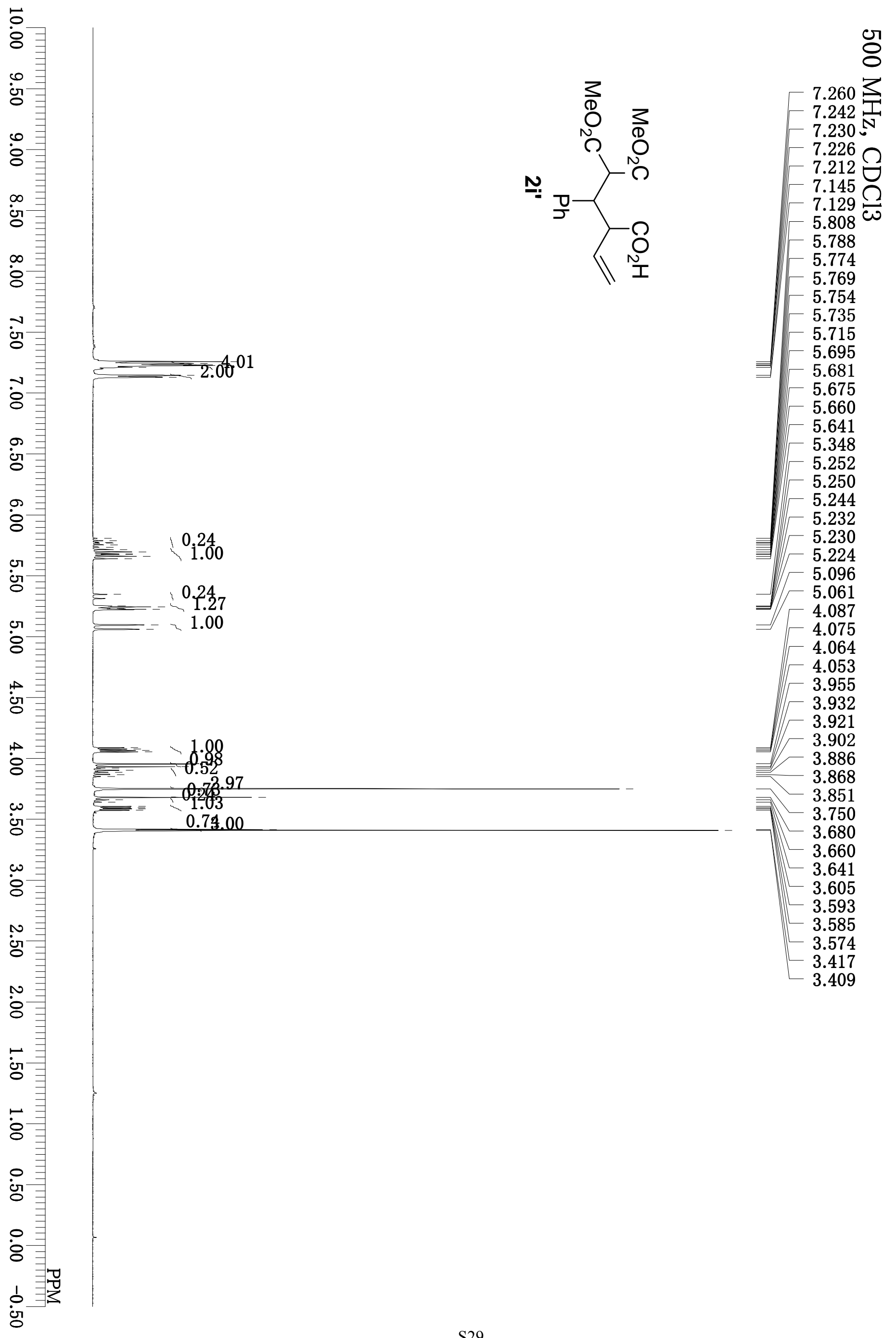




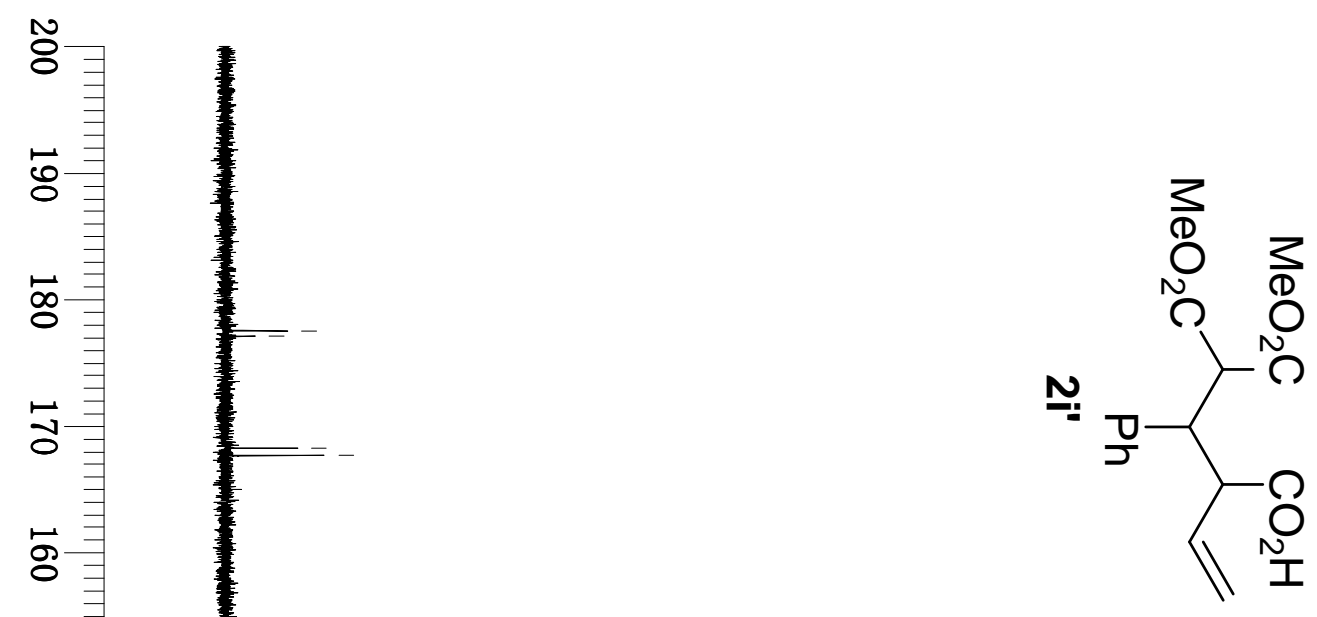

136.405

133.438

131.740

129.060

128.822

128.278

128.192

127.658

127.610

120.409

o

ธ요

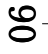

$\infty$

77.258

77.000

76.742

ฮै

용

용

政

。

ธิ

후

o

光

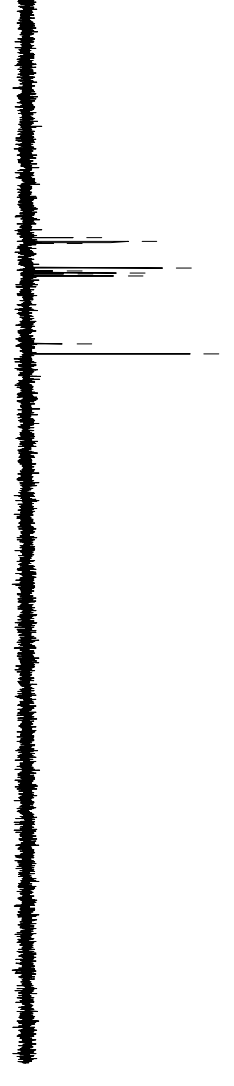

55.157

54.833

54.728

52.801

52.544

52.410

52.305

52.134

46.811

45.991 


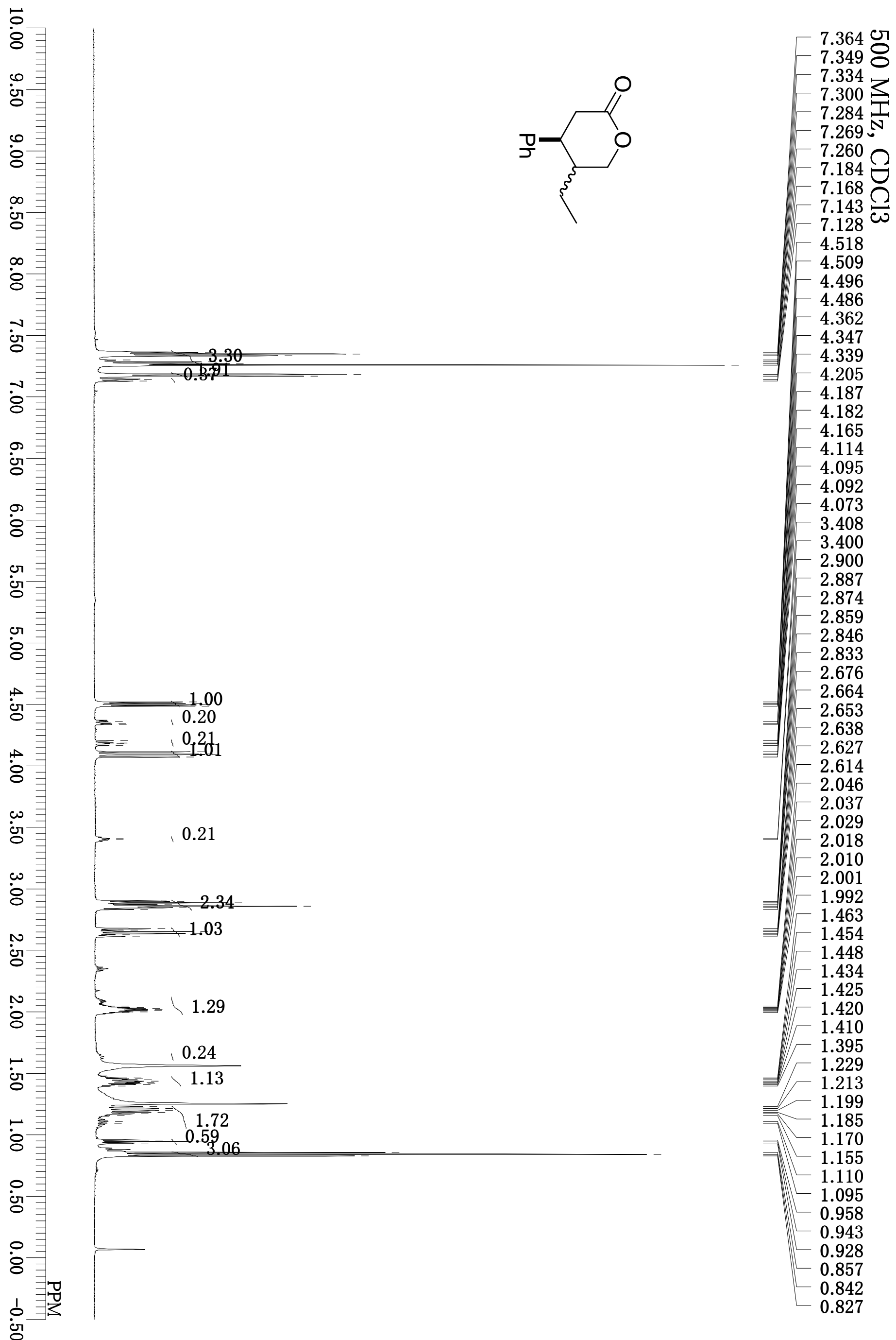




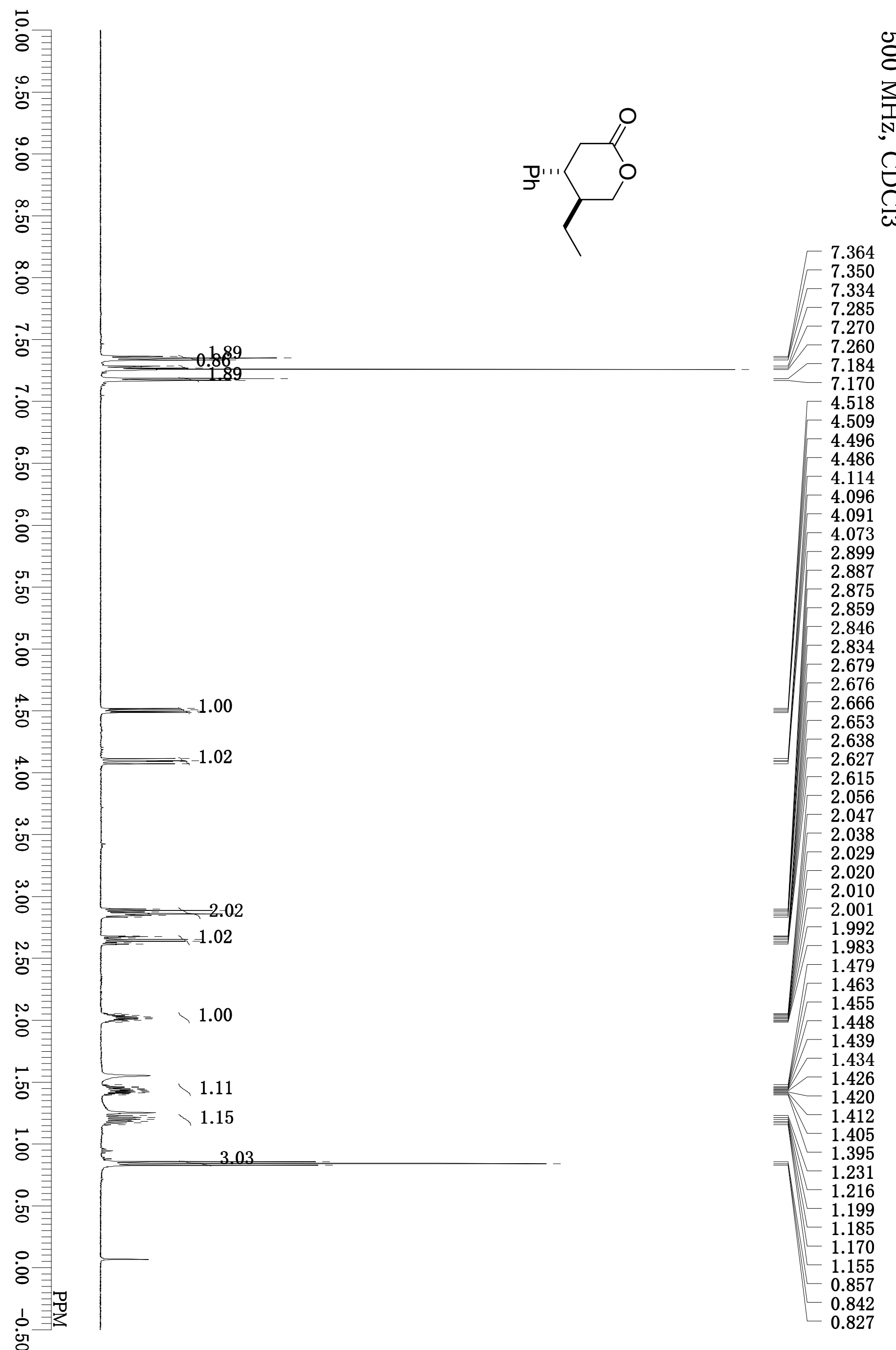



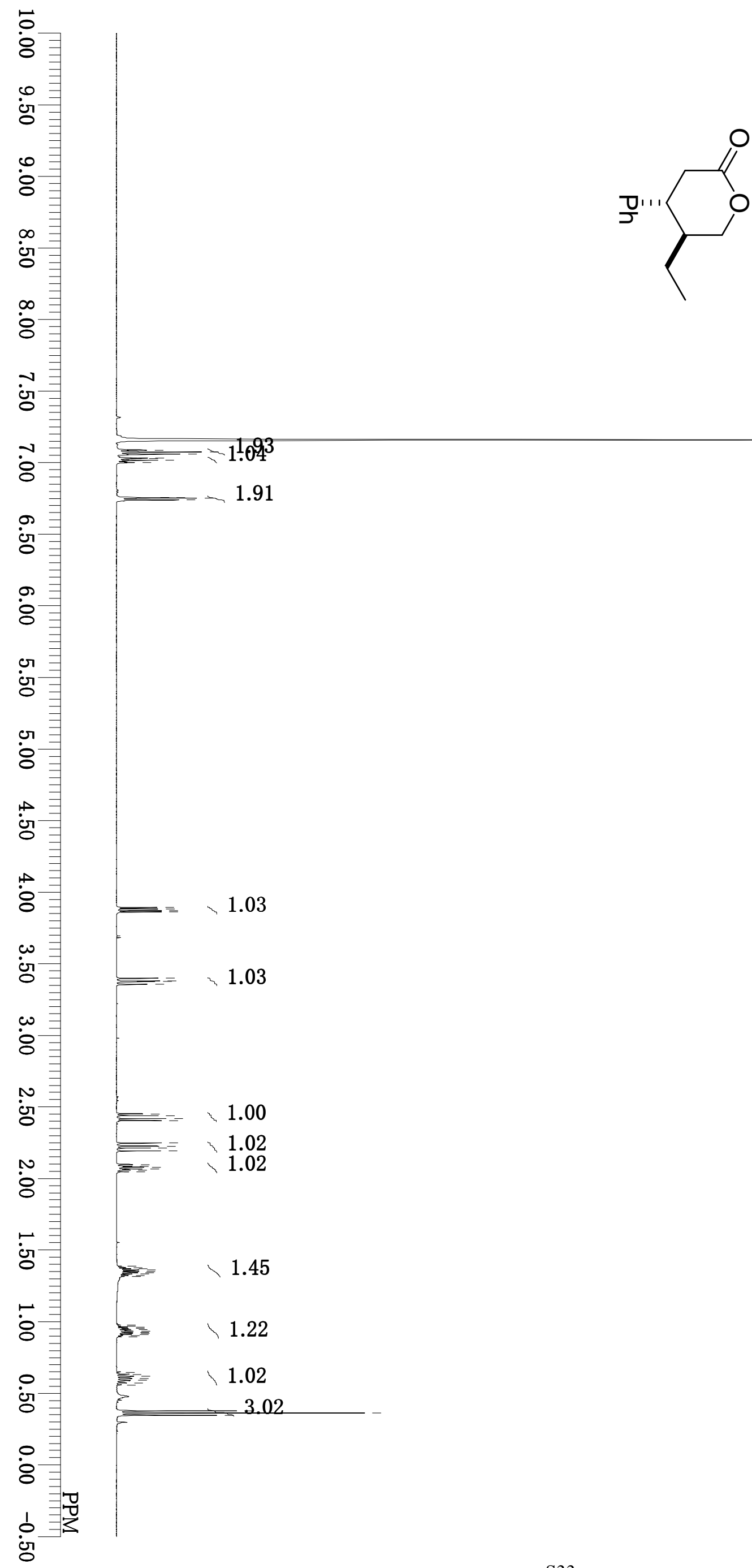


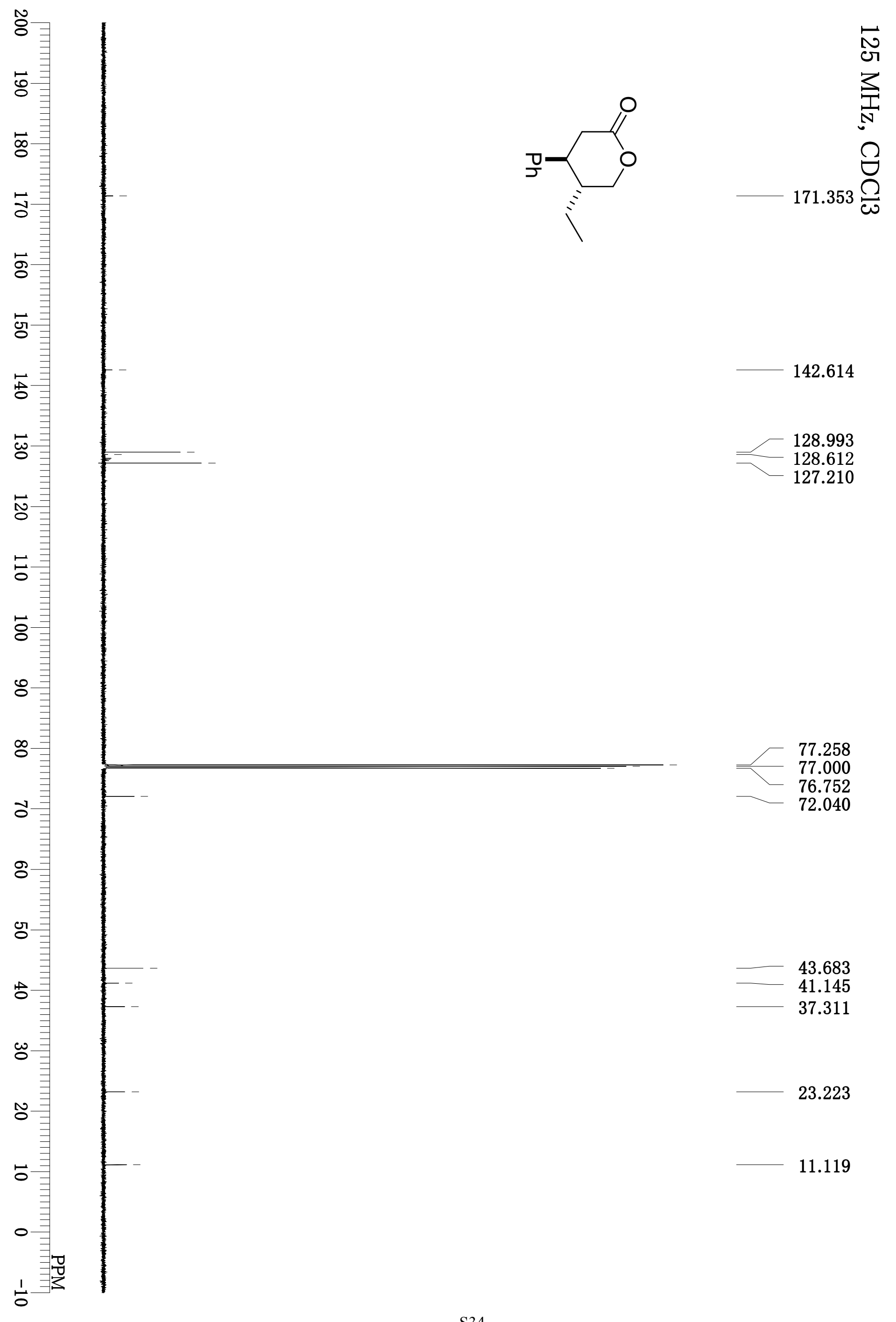

